\title{
COMPLEX INTERPOLATION FOR \\ NORMED AND QUASI-NORMED SPACES \\ IN SEVERAL DIMENSIONS. III. REGULARITY RESULTS FOR HARMONIC INTERPOLATION
}

\author{
ZBIGNIEW SLODKOWSKI
}

\begin{abstract}
The paper continues the study of one of the complex interpolation methods for families of finite-dimensional normed spaces $\left\{\mathbf{C}^{n},\|\cdot\|_{z}\right\}_{z \in G}$, where $G$ is open and bounded in $\mathbf{C}^{k}$. The main result asserts that (under a mild assumption on the datum) the norm function $(z, w) \rightarrow\|w\|_{z}^{2}$ belongs to some anisotropic Sobolew class and is characterized by a nonlinear PDE of second order. The proof uses the duality theorem for the harmonic interpolation method (obtained earlier by the author). A new, simpler. proof of this duality relation is also presented in the paper.
\end{abstract}

\section{INTRODUCTION}

This paper is a continuation of $[17,19]$. As in those papers, we study interpolation families of finite-dimensional normed spaces of the form $\left\{\mathbf{C}^{n},\|\cdot\|_{z}\right\}_{z \in G}$, where $G$ is an open subset of $\mathbf{C}^{k}$. To set our results in perspective, we start with some historical comments.

The basis work of Coifman et al. [3] dealt with the case of $G=$ the unit disc. Rochberg reinterpreted and expanded their results using a notion of curvature for complex Finsler bundles, due to Kobayashi [21]. He has shown, among other things (see Rochberg [12], Rochberg and Weiss [13]), that interpolation families [3] are characterized by a vanishing curvature condition which is a relation between second-order derivatives of $f(z, w)=\frac{1}{2}\|w\|_{z}^{2}$. If the norms are defined by inner product, i.e., $\|w\|_{z}^{2}=\langle\Omega(z) w, \bar{w}\rangle$, where $\Omega(z)$ is an $n \times n$ matrix, $z \in G$, the characterization takes the particularly simple form $\bar{\partial}\left(\Omega(z)^{-1} \partial \Omega(z)\right)=0$. Coifman has proposed the following generalization of this equation to higher dimensions:

$$
\sum_{i=1}^{k} \bar{\partial}_{i}\left(\Omega(z)^{-1} \partial_{i} \Omega(z)\right)=0, \quad z=\left(z_{1}, \ldots, z_{k}\right) \in \Omega \subset \mathbf{C}^{k}
$$

Received by the editors November 3, 1988.

1980 Mathematics Subject Classification (1985 Revision). Primary 32F05; Secondary 31B35, 35J60, 32L15.

Key words and phrases. Plurisubharmonic functions, Hessian form, weak derivative, dual norm, interpolation family, harmonic multifunction.

This research was supported by National Science Foundation grant DMS-870227. 
He considered this condition as a right way to define interpolation families over $G \subset \mathbf{C}^{k}$ in case of inner product spaces and posed the problem of developing a general interpolation method extending this idea. However, it remained open for some time.

In the winter and spring of 1985 , the author constructed, in the case of a strictly pseudoconvex domain $G$ in $\mathbf{C}^{k}, k$ distinct interpolation methods by means of the Perron procedure applied to some families of set-valued functions; see [14a, b; 17, §7]. (This construction was also new in the case of a planar domain $G$.) Expanding this approach in the winter and spring of 1986, the author obtained a variety of interpolation methods (see [14c, 17]), one of which, called harmonic interpolation, is consistent with condition $(0.0)$ in the case of inner product norms $\|w\|_{z}^{2}=\langle\Omega(z) w, \bar{w}\rangle$. For further information on harmonic interpolation see [17, §8] and [19]. The harmonic interpolation method has been independently obtained and studied by Coifman and Semmes [5], who have also solved (among many other things) equation (0.0) by PDE techniques.

A harmonic interpolation family $\left(G,\|\cdot\|_{z}\right)_{z \in G}$ can be characterized by a differential equation resembling $(0.0)$ even if the norms are non-Hilbertian. Consider, for any $(z, w) \in G \times \mathbf{C}^{n}$, the complex Hessian form of $f(z, w)=\frac{1}{2}\|w\|_{z}^{2}$ at $(z, w)$. The coefficient matrix of this form, with respect to the coordinate system $z_{1}, z_{2}, \ldots, z_{k} ; w_{1}, \ldots, w_{n}$ is naturally divided into blocks $H_{11}$, $H_{12}, H_{21}, H_{22}$. In case the norm function $f(z, w)$ has classical derivatives at a point $\left(z_{0}, w_{0}\right)$ and $H_{22}$ is positive definite, then

$$
\operatorname{tr}\left(H_{11}-H_{12} H_{22}^{-1} H_{21}\right)=0
$$

(at this point). Otherwise, condition $(0.1)$ can be interpreted as holding in a certain, rather weak, sense; see $[17,18]$.

Equation $(0.1)$ is a nonlinear degenerate elliptic equation and, at present, there seems to be no general theory with which to treat it. Equation $(0.1)$ reduces to $(0.0)$ in the case of inner product norms $\|w\|_{z}^{2}=\langle\Omega(z) w, \bar{w}\rangle$. Explicit solutions of $(0.1)$ are rare. If $n=1$, then $\|w\|_{z}^{2}=\omega(z)|w|^{2}$ and $(0.1)$ is equivalent to $\Delta(\log \omega)=0$. If the boundary norms are $l^{p}$, say $\|w\|_{\zeta}=$ $\left(\sum_{j=1}^{n}\left|w_{j}\right|^{p(\zeta)}\right)^{1 / p(\zeta)}, \zeta \in \partial G$, then the solution is $\|w\|_{z}=\left(\sum_{j=1}^{n}\left|w_{j}\right|^{\tilde{p}(z)}\right)^{1 / \hat{p}(z)}$, $z \in G$, where $1 / \tilde{p}(z)$ is the harmonic extension of $1 / p(\zeta)$ to $G[5,11,19]$. In case of one variable $(k=1)$, equation (0.1) is equivalent to Rochberg's vanishing curvature condition mentioned above (some work is needed to check this); in fact (for $k \geq 1$ ), the matrix $H_{11}-H_{12} H_{22}^{-1} H_{21}$ is closely related to the Kobayashi curvature form of the Finsler bundle $\left(G \times \mathbf{C}^{n},\|w\|_{z}^{2}\right)$ [21].

The purpose of this paper is to show that, under mild regularity properties of the boundary data, the harmonic interpolation family satisfies condition $(0.1)$ in the usual sense. We also present a simpler proof of the duality theorem for harmonic interpolation (obtained earlier by the author in $[17, \S 6]$ ). 
We will now review definitions and results related to the harmonic interpolation method which will be used below, and then formulate the main results of this paper.

Definition 0.1. Let $U \subset \mathbf{C}^{k} \times \mathbf{C}^{n}$ be open. We say that $u \in P_{\text {subh }}(U)$ if $u(z, w)$ is an usc (= upper semicontinuous) function on $U$ and for every holomorphic function $z \rightarrow f(z): G \rightarrow \mathbf{C}^{n}, G \subset \mathbf{C}^{k}$, the function $z \rightarrow u(z, f(z))$ is subharmonic in $\{z:(z, f(z)) \in G\}$.

Remark. It is enough to consider in this definition only $\mathbf{C}$-affine functions.

Definition 0.2. An usc compact-valued function $z \rightarrow K(z): G \rightarrow 2^{\mathrm{C}^{n}}$ (briefly, multifunction) is called harmonic if, for every compact set $B$ such that $B \cap$ $X$ is compact, where $X=\operatorname{gr}(K)=\{(z, w): z \in G, w \in K(z)\}$, and for every function $u(z, w)$ which is $P_{\text {subh }}$ near $B$, it holds that $\max u \mid B \cap X \leq$ $\max u \mid(\partial B) \cap X$.

Theorem 0.3. Let $G \subset \mathbf{C}^{k}$ be open, bounded, and regular with respect to the usual Dirichlet problem. Let $\left\{p_{\zeta}(\cdot)\right\}_{\zeta \in \partial G}$ be a continuous family of norms on $\mathbf{C}^{n}$, with $p_{\zeta}\left(e^{i \theta} w\right)=p_{\zeta}(w), \theta \in R, w \in \mathbf{C}^{n}$. Denote $B(\zeta)=\left\{w \in \mathbf{C}^{n}: p_{\zeta}(w) \leq 1\right\}$ and define $z \rightarrow W(z): \bar{G} \rightarrow 2^{\mathrm{C}^{n}}$ as the union of all usc multifunctions $K: \bar{G} \rightarrow 2^{\mathrm{C}^{n}}$ which are harmonic on $G$ and such that $K(\zeta) \subset W(\zeta), \zeta \in \partial G$. Then

(a) $z \rightarrow W(z): \bar{G} \rightarrow 2^{\mathbf{C}^{n}}$ is a continuous family of compact, convex, circled sets such that $W \mid \partial G=B$;

(b) $W(\cdot)$ is a harmonic multifunction on $G$;

(c) if $\tilde{p}_{z}(\cdot)$ is the Minkowski function of $W(z)$, then the function $(z, w) \rightarrow$ $\log \tilde{p}_{z}(w)$ is continuous and $P_{\text {subh }}$ on $G \times \mathbf{C}^{n}$.

Definition 0.4. The unique family of normed spaces $\left\{\mathbf{C}^{n}, \tilde{p}_{z}(\cdot)\right\}_{z \in \bar{G}}$ described in the above theorem is called the harmonic interpolation family extending (corresponding to) the given family $\left\{\mathbf{C}^{n}, p_{\zeta}(\cdot)\right\}_{\zeta \in \partial G}$.

See $[17, \S \S 1,8 ; 19 ; 3 ; 11]$ for background and further information.

We now formulate two characterizations of harmonic interpolation families, which we will use below.

Characterization 0.5. Let $\left\{\mathbf{C}^{n}, p_{z}(\cdot)\right\}_{z \in \bar{G}}, G \subset \mathbf{C}^{k}$, be a continuous family of normed (or quasinormed) spaces. Then it is a harmonic interpolation family if and only if the function $(z, w) \rightarrow \log p_{z}(w)$ lies in $P_{\text {subh }}\left(G \times \mathbf{C}^{n}\right)$ and $z \rightarrow$ $W(z): G \rightarrow 2^{\mathrm{C}^{n}}$ is a harmonic multifunction, where $W(z)=\left\{w: p_{z}(w) \leq 1\right\}$.

This is a conclusion from [17, Remark 3.4 and Theorems 3.5, 4.1].

Characterization 0.6. Let $\left\{\mathbf{C}^{n},\|\cdot\|_{z}\right\}_{z \in \bar{G}}$ be a continuous family of normed spaces. Then it is a harmonic interpolation family if and only if both $f(z, w)=$ $\frac{1}{2}\|w\|_{z}^{2},(z, w) \in G \times \mathbf{C}^{n}$, and $g(z, \xi)=\frac{1}{2}|\xi|_{z}^{2},(z, \xi) \in G \times \mathbf{C}^{n}$, are functions of class $P_{\text {subh }}$, where $|\cdot|_{z}$ denotes the dual norm to $\|\cdot\|_{z}$.

The proof is given at the end of $\S 2$. 
One of the main results of [17, Theorem 6.1] was a duality theorem for a class of complex interpolation methods. Specified to the harmonic interpolation case, it reads as follows.

Theorem 0.7. Let $\left\{\mathbf{C}^{n},\|\cdot\|_{z}\right\}_{z \in \bar{G}}$ be a harmonic interpolation family of normed spaces. Then the dual normed spaces $\left\{\mathbf{C}^{n},|\cdot|_{z}\right\}_{z \in \bar{G}}$ form a harmonic interpolation family.

In $\S 2$ we present a new, direct proof of this fact, considerably simpler than the general one given in $[17, \S 2]$. It is based on elementary analysis of quadratic forms, carried out in $\S 1$.

The major part of the paper is devoted to the proof of regularity results, Theorems 0.10 and 0.11 . Their setup is motivated by the following observation from [19, Corollary 2.11, Theorem 2.12].

Property 0.8. Let $\left\{\mathbf{C}^{n},\|\cdot\|_{z}\right\}_{z \in \bar{G}}$ be a harmonic interpolation family, with $G$ bounded. Assume that the boundary norms are uniformly convex and uniformly smooth, in the following sense:

$$
\begin{aligned}
2 f_{\zeta}(w)+2 \varepsilon|h|^{2} \leq f_{\zeta}(w+h)+f_{\zeta}(w-h) \leq & 2 f_{\zeta}(w)+2 K|h|^{2}, \\
& \zeta \in \partial G, w, h \in \mathbf{C}^{n},
\end{aligned}
$$

with $\varepsilon>0, K<+\infty$ independent of $\zeta$, where $f_{z}(w)=\frac{1}{2}\|w\|_{z}^{2}, z \in \bar{G}$. Then $f_{z}(\cdot), z \in G$, satisfy condition $(0.2)$ with the same constants.

Notation 0.9. If $f(z, w)$ is an $L_{\text {loc }}^{1}$ function on a subset of $\mathbf{C}^{k} \times \mathbf{C}^{n}$, we denote by $H_{11} f, H_{12} f, H_{21} f$, and $H_{22} f$ the matrix-valued distributions $\left(\partial^{2} f / \partial \bar{z}_{i} \partial \bar{z}_{j}\right)_{i j}, \quad\left(\partial^{2} f / \partial \bar{z}_{i} \partial w_{q}\right)_{i q}, \quad\left(\partial^{2} f / \partial \bar{w}_{p} \partial z_{j}\right)_{p j}$, and $\left(\partial^{2} f / \partial \bar{w}_{p} \partial w_{q}\right)_{p q}$ (which are "blocks" of the complex Hessian of $f$, of sizes $k \times k, k \times n$, $n \times k$, and $n \times n$, respectively). In case the distribution $H_{i j} f$ is represented by an $L_{\text {loc }}^{1}$ (matrix-valued) function, we denote the corresponding function by $H_{i, j} f(z, w)$, or simply by $H_{i j} f$.

Theorem 0.10. Let $\left\{\mathbf{C}^{n},\|\cdot\|_{z}\right\}_{z \in \bar{G}}$ be a harmonic interpolation family, with boundary norms $\|\cdot\|_{\zeta}, \zeta \in \partial G$, uniformly convex and uniformly smooth as in Property 0.8. Denote $H_{i j}=H_{i j} f, i, j=1,2$, where $f(z, w)=\frac{1}{2}\|w\|_{z}^{2}$. Then $H_{22}, H_{22}^{-1} \in L^{\infty}, H_{12}, H_{21} \in L_{\mathrm{loc}}^{2}$, and $\operatorname{tr} H_{11} \in L_{\mathrm{loc}}^{1}$, on $G \times \mathbf{C}^{n}$, and

$$
\operatorname{tr} H_{11}-\operatorname{tr} H_{12} H_{22}^{-1} H_{21}=0 \text { a.e. on } G \times \mathbf{C}^{n} \text {. }
$$

The next theorem is, in a sense, a converse result.

Theorem 0.11. In the notation of the last theorem, if $\left\{\mathbf{C}^{n},\|\cdot\|_{z}\right\}_{z \in \bar{G}}$ is a continuous family of normed spaces such that $H_{22}, H_{22}^{-1} \in L_{\mathrm{loc}}^{\infty}\left(G \times \mathbf{C}^{n}\right), H_{12}$, $H_{21} \in L_{\mathrm{loc}}^{2}\left(G \times \mathbf{C}^{n}\right)$, $\operatorname{tr} H_{11} \in L_{\mathrm{loc}}^{1}\left(G \times \mathbf{C}^{n}\right)$, and (0.3) holds a.e. in $G \times \mathbf{C}^{n}$, then spaces $\left\{\mathbf{C}^{n},\|\cdot\|_{z}\right\}_{z \in \bar{G}}$ form a harmonic interpolation family.

The essential idea of the proof of both theorems consists in showing that the quantity $(0.3)$ computed for the dual function $\frac{1}{2}\|\cdot\|_{z}^{2}$ is equal to the negative 
of (0.3) (composed with the gradient mapping). Then Characterization 0.6 is used. The main argument is carried out in $\S 4$. The computation of the real Hessian of the norm function relies on some delicate forms of chain rule, in particular, on the following inverse function lemma (proved in $\S 5$ ).

Lemma 0.12. Let $G: \Omega \times R^{n} \rightarrow \Omega \times R^{n}$, where $\Omega \subset R^{k}$ is open, be a homeomorphism, with $G(x, y)=\left(x, G_{x}(y)\right)$. Denote $F=G^{-1}$. Assume that the maps $G_{x}: R^{n} \rightarrow R^{n}$ are locally uniformly (in $x, y$ ) bi-Lipschitz, and that $\partial G / \partial x_{i} \in L_{\mathrm{loc}}^{2}\left(\Omega \times R^{n}\right)$ (as distributions). Then the distributions $\partial F / \partial x_{i}$, $i=1, \ldots, k$, and $\partial F / \partial y_{j}, j=1, \ldots, n$, lie in $L_{\mathrm{loc}}^{2}$ and $L_{\mathrm{loc}}^{\infty}$, respectively, and for a.a. $(x, y) \in \Omega \times R^{n}$ the Jacobian matrix of $F$ at $(x, y)$ is equal to the inverse of the Jacobian matrix of $G$ at $F(x, y)$.

Finally, the information on the dual complex Hessian is extracted from that on the real Hessian through an algebraic formalism described in $\S 3$.

\section{DECOMPOSITION OF A SYMMETRIC BILINEAR FORM RELATIVE TO A PROJECTION}

The essential part of the proof of duality for harmonic interpolation families relies on the following fact.

Lemma 1.1. Let $M$ be an open subset of $\mathbf{C}^{k}$ and $u: M \times \mathbf{C}^{n} \times \mathbf{C}^{m} \rightarrow R$ be a $\mathbf{C}^{(2)}$ smooth function. Define $v(z, \xi)=\inf _{w} u(z, \xi, w),(z, \xi) \in M \times \mathbf{C}^{n}, w \in \mathbf{C}^{m}$. Assume that $m \leq n$ and that for every $(z, \xi) \in M \times \mathbf{C}^{n}$, the slice function $w \rightarrow u(z, \xi, w)$ is strongly convex. Fix $\left(z_{0}, \xi_{0}, w_{0}\right) \in M \times \mathbf{C}^{n} \times \mathbf{C}^{m}$ and denote by $H_{u}$ and $H_{v}$ the complex Hessian forms of $u$ and $v$ at $\left(z_{0}, \xi_{0}, w_{0}\right)$ and $\left(z_{0}, \xi_{0}\right)$, respectively. Then $v$ is $\mathbf{C}^{(2)}$-smooth and either $H_{v}$ is degenerate in some direction $(0,0) \neq\left(0, \xi_{1}\right) \in\{0\} \times \mathbf{C}^{n}$ (i.e., $H_{v}\left(0 \oplus \xi_{1}, 0 \oplus \xi_{1}\right)=0$ ) or there exist $\mathbf{C}$-linear maps $S_{0}: \mathbf{C}^{k} \rightarrow \mathbf{C}^{n}$ and $S=\left(S_{0}, S_{1}\right): \mathbf{C}^{k} \rightarrow \mathbf{C}^{n} \times \mathbf{C}^{m}$, such that for every vector $z \in \mathbf{C}^{k}$

$$
H_{v}\left(z \oplus S_{0} z, z \oplus S_{0} z\right)=H_{u}(z \oplus S z, z \oplus S z) .
$$

For the proof (which is given at the end of this section) we have to relate the Hessian forms of $u$ and $v$. To facilitate this, as well as other instances of dealing with Hessian forms, we introduce the notion of decomposition of a quadratic form, which we discuss first.

Definition 1.2. (a) Let $E, Z$ be finite-dimensional real vector spaces, $\pi: E \rightarrow$ $Z$ an epimorphism, and $b: E \times E \rightarrow R$ a symmetric bilinear form. Denote $W=\operatorname{ker} \pi$. A representation

$$
b(x, x)=b_{0}(\pi x, \pi x)+b_{1}(x, x)
$$

is called a canonical decomposition of $b$ relative to $\pi$ if $b_{0}: Z \times Z \rightarrow R$ and $b_{1}: E \times E \rightarrow R$ are symmetric bilinear forms and there exists a linear map $\rho: E \rightarrow W$ such that $\rho \mid W=\mathrm{id}_{W}$ and $b_{1}(x, x)=b(\rho x, \rho x)$. 
(b) By the next lemma, if a canonical decomposition relative to $\pi$ exists, it is unique. In such an instance, the unique form $b_{0}$ is called the projection of $b$ under $\pi$ and denoted $\pi b$.

Lemma 1.3. In the situation of Definition 1.2, denote by $D$ and $D_{W}$ the degeneracy spaces of the forms $b$ and $b \mid W \times W$, respectively, i.e., $D=\left\{x_{0}: b\left(x_{0}, x\right)=\right.$ $0, x \in E\}, D_{w}=\left\{w_{0}: b\left(w_{0}, w\right)=0\right.$ for $\left.w \in W\right\}$. Then a canonical decomposition exists if and only if $D_{W} \subset D$. Furthermore, if $D_{W} \subset D$, then

(a) $N \cap W=D_{W}=D \cap W, N+W=E$, where $N=\{x \in E: b(x, w)=0$ for $w \in W\}$;

(b) there is a linear map $s: Z \rightarrow N$, such that $\pi s=i d_{Z}$;

(c) the forms

$$
b_{0}(z, z)=b(s z, s z), \quad z \in Z,
$$

and

$$
b_{1}(x, x)=b((I-s \pi) x,(I-s \pi) x), \quad x \in E,
$$

are independent of the choice of $s$ satisfying (b) and

$$
b(x, x)=b_{0}(\pi x, \pi x)+b_{1}(x, x), \quad x \in E ;
$$

(d) the representation (1.2) is the unique canonical decomposition.

Proof. If a canonical decomposition (1.2) exists, we get by polarization $b\left(w_{0}, x\right)$ $=b_{0}\left(\pi w_{0}, \pi x\right)+b\left(\rho w_{0}, \rho x\right)=0+b\left(w_{0}, \rho x\right)$, if $w_{0} \in W$. If $w_{0} \in D_{W}$, then $b\left(w_{0}, \rho x\right)=0$, because $\rho x \in W$. Hence, the condition $D_{W} \subset D$ is necessary. Its sufficiency will follow from parts (a) through (d). We assume $D_{W} \subset D$ for the rest of the proof.

(a) The relations $N \cap W=D_{W}$ and $D \cap W \subset D_{W}$ are obvious. Hence, $D \cap W=D_{W}=N \cap W$. If $\widetilde{N}, \widetilde{W}, \widetilde{E}$ denote respectively the quotient spaces $N / D_{W}, W / D_{W}, E / D_{W}$, then $\widetilde{N} \cap \widetilde{W}=[0]$. Since $D_{W}$ is contained in the degeneracy space of $b$, the latter defines a symmetric bilinear form $\tilde{b}: \widetilde{E} \times$ $\widetilde{E} \rightarrow R$ (where $\left.\tilde{b}\left(x+D_{W}, y+D_{W}\right)=b(x, y)\right)$. It is clear that $\widetilde{N}=\{[x] \in$ $\widetilde{E}: \tilde{b}([x],[y])=0$ for $[y] \in \widetilde{E}\}$. Since $\widetilde{N} \cap \widetilde{W}=[0]$, the form $\tilde{b} \mid \widetilde{W} \times \widetilde{W}$ is nondegenerate and so $\operatorname{dim} \widetilde{N}=\operatorname{dim} \widetilde{E}-\operatorname{dim} \widetilde{W}$, and consequently $\widetilde{N}+\widetilde{W}=\widetilde{E}$. Thus $N+W=E$.

(b) Since $\pi(N)=\pi(N+W)=\pi(E)=Z$, the existence of $s: Z \rightarrow N$ with $\pi s=\mathrm{id}_{Z}$ is obvious.

(c) The independence of $b_{0}, b_{1}$ of the choice of $s$ follows from (d). Furthermore,

$$
\begin{aligned}
b(x, x) & =b(s \pi x+(I-s \pi) x, s \pi x+(I-s \pi) x) \\
& =b(s \pi x, s \pi x)+b((I-s \pi) x,(I-s \pi) x)+2 b(s \pi x,(I-S \pi) x) \\
& =b_{0}(\pi x, \pi x)+b_{1}(x, x)
\end{aligned}
$$

for $b(s \pi x,(I-s \pi) x)=0$, since $s \pi x \in N$ and $(I-s \pi) x \in \operatorname{ker} \pi=W$.

(d) Let (1.2) be a canonical decomposition of $b$ and let $D_{1}=\left\{x_{0} \in E\right.$ : $b_{1}\left(x_{0}, x\right)=0$, for $\left.x \in E\right\}$ (the degeneracy space of $b_{1}$ ). If $x_{0} \in D_{1}$ and 
$w \in W$, then $b\left(x_{0}, w\right)=b_{0}\left(\pi x_{0}, \pi w\right)+b_{1}\left(x_{0}, w\right)=0$ and so $D_{1} \subset N$. Clearly, $\operatorname{ker} \rho \subset D_{1}$, and since $\rho \mid W=\mathrm{id}_{W}, \operatorname{ker} \rho+W=E$. Hence, $D_{1}+W=$ $E$. Furthermore, $D_{1} \cap W=D \cap W$ because, if $w_{0} \in W$, then $b_{1}\left(w_{0}, x\right)=$ $b\left(w_{0}, \rho x\right)$ and $\rho x \in W$. Since $D \cap W=N \cap W$ (by (a)), we obtain that $D_{1} \subset N, D_{1}+W=N+W$, and $D_{1} \cap W=N \cap W$. Thus $D_{1}=N$. If we now choose any map $s: Z \rightarrow N$ satisfying condition (b), we get $b_{1}(s z, s z)=0$, and so $b(s x, s x)=b_{0}(z, z)$. This proves the uniqueness of $b_{0}$ (and so of $b_{1}$ ), as well as its independence of the choice of $s$. Q.E.D.

Corollary 1.4. If, in the situation of Definition 1.2, the restriction of the form $b$ to $W$ is nondegenerate (i.e., $D_{W}=(0)$ ), then the canonical decomposition of $b$ relative to $\pi$ exists, the map $s: Z \rightarrow N$ such that $\pi s=\mathrm{id}_{Z}$ is unique, and

$$
(\pi b)(z, z)=b_{0}(z, z)=b(s z, s z) .
$$

Furthermore, for every $z \in Z, s z$ is the unique critical point of the function $x \rightarrow b(x, x): \pi^{-1}(z) \rightarrow R$.

Proof. By Lemma 1.3(a), if $D_{W}=(0)$, then $N \cap W=(0)$ and the uniqueness of $s$ is obvious, namely

$$
N \cap \pi^{-1}(z)=\{s(z)\}, \quad \text { for } z \in Z .
$$

Fix $z \in Z$ and $x_{0} \in \pi^{-1}(z)$. If we parametrize $\pi^{-1}(z)$ by $x=x_{0}+w$, $w \in W$, then $b(x, x)=b\left(x_{0}, x_{0}\right)+2 b\left(x_{0}, w\right)+b(w, w)$, and the gradient of $b \mid \pi^{-1}(z)$ is the linear form $w \rightarrow 2 b\left(x_{0}, w\right): W \rightarrow R$, which vanishes identically if and only if $x_{0} \in N$. By this and (1.5), $x_{0}$ is a critical point if and only if $x_{0}=s(z)$. Q.E.D.

The next observation, which follows directly from the last corollary, hints at a connection between Lemma 1.1 and the operation of projection defined above.

Remark 1.5. With $E, Z, \pi, b$ as in Definition 1.2, assume, in addition, that the restriction of the form $b$ to $\operatorname{ker} \pi$ is positive definite. Then

$$
(\pi b)(z, z)=\inf \left\{b(x, x): x \in \pi^{-1}(z)\right\}, \quad z \in Z .
$$

Proposition 1.6. Let $E=Z \oplus W, \pi(z \oplus w)=z,(z \oplus w) \in E$. Let $b: E \times E \rightarrow R$ be a symmetric bilinear form, whose block representation with respect to the decomposition $E=Z \oplus W$ is

$$
b\left(x, x^{\prime}\right)=\left\langle B_{11} z, z^{\prime}\right\rangle+\left\langle B_{12} w, z^{\prime}\right\rangle+\left\langle B_{21} z, w^{\prime}\right\rangle+\left\langle B_{22} w, w^{\prime}\right\rangle,
$$

where $x=z \oplus w, x^{\prime}=z^{\prime} \oplus w^{\prime}, B_{11}: Z \rightarrow Z^{*}, B_{12}: W \rightarrow Z^{*}, B_{21}: Z \rightarrow W^{*}$, $B_{22}: W \rightarrow W^{*}$, and $B_{i j}^{T}=B_{j i}, i=1,2$. ( $B^{T}$ denotes the dual or transpose operator.) Then $b$ is nondegenerate on $\operatorname{ker} \pi$ if and only if $B_{22}$ is nonsingular. If $B_{22}$ is nonsingular, then the projection of $b$ under $\pi$ is

$$
(\pi b)(z, z)=\left\langle\left(B_{11}-B_{12} B_{22}^{-1} B_{21}\right) z, z\right\rangle, \quad z \in Z .
$$


Furthermore, $s(z)=z \oplus\left(-B_{22}^{-1} B_{21} z\right), z \in Z$, and the canonical decomposition of $b$ relative to $\pi$ is

$$
b(x, x)=(\pi b)(z, z)+\left\langle B_{22}\left(w+B_{22}^{-1} B_{21} z\right), w+B_{22}^{-1} B_{21} z\right\rangle .
$$

Proof (sketch). Letting $x^{\prime}=0 \oplus w^{\prime}, x=z \oplus w$ in (1.6), we get $b\left(x, w^{\prime}\right)=$ $\left\langle B_{21} z+B_{22} w, w^{\prime}\right\rangle$, and so $x \in N$ (see Lemma 1.3(a)), if and only if $B_{21} z+$ $B_{22} w=0$. By this and Lemma 1.3(b), $s z=z \oplus\left(-B_{22}^{-1} B_{21} z\right)$. The remaining statements then follow from Lemma 1.3(c). Q.E.D.

Remark 1.7. Let $u(x, y)$ be a $\mathbf{C}^{(2)}$-smooth function on $D \times R^{n}$, where $D \subset R^{k}$ is open. Assume that $u$ is strongly convex on every hyperplane $\{x\} \times R^{n}$, $x \in D$. Define $v(x)=\inf \left\{u(x, y): y \in R^{n}\right\}, x \in D$. Then $v$ is $\mathbf{C}^{(2)}$-smooth in $D$ and, for every $a \in D$, the Hessian form of $v$ at $a$ is the projection under $\pi$ of the Hessian form of $u$ at $(a, b)$, where $\pi: R^{k} \times R^{n} \rightarrow R^{k}$ is the standard projection map and $b$ denotes the unique point, such that $u(a, b)=v(a)$.

(This is a reinterpretation of a well-known consequence of the implicit function theorem [17, equation (5.1)] in terms of Proposition 1.6, in particular equation (1.7).)

Proof of Lemma 1.1. Let $E=\mathbf{C}^{k} \oplus \mathbf{C}^{n} \oplus \mathbf{C}^{m}, Z=\mathbf{C}^{k} \oplus \mathbf{C}^{n}$, and let $\pi: E \rightarrow Z$ be defined by $\pi(x)=z \oplus \xi$, for $x=(z \oplus \xi \oplus w) \in E$. Let $b: E \times E \rightarrow R$ be the real Hessian form of $u$ at $\left(z_{0}, \xi_{0}, w_{0}\right)$. Since the function $u(z, \xi, w)$ is strongly convex in $w$, the restriction of the form $b$ to $W=\operatorname{ker} \pi=(0) \oplus(0) \oplus \mathbf{C}^{m}$ is nondegenerate. Spaces $E$ and $Z$ also have a complex structure and we let $J: E \rightarrow E$ and $J: Z \rightarrow Z$ denote the operators of multiplication by $i$, so that $J^{2}=-\mathrm{id}$. Let $h(\cdot, \cdot)$ and $a(\cdot, \cdot)$ denote, respectively, the Hermitian and anti-Hermitian parts of $b(\cdot, \cdot)$, i.e., $b(x, x)=h(x, x)+a(x, x), h(J x, J x)=$ $h(x, x), a(J x, J x)=-a(x, x), x \in E$.

Let $N \subset E$ and $s: Z \rightarrow N$ be defined as in Lemma 1.3. Since $b$ is nondegenerate on $W, s(Z)=N$ (see Corollary 1.4). Denote by $N_{h}$ and $N_{a}$ the annihilators of $W=\operatorname{ker} \pi$ relative to $h$ and $a$, respectively, i.e.,

$$
\begin{aligned}
& N_{h}=\{x \in E: h(x, w)=0 \text { for } w \in W\}, \\
& N_{a}=\{x \in E: a(x, w)=0 \text { for } w \in W\} .
\end{aligned}
$$

Since the forms $h$ and $a$ are, respectively, Hermitian and anti-Hermitian and $J(W)=W$, both $N_{h}$ and $N_{a}$ must be complex subspaces of $E$ with

$$
\operatorname{codim}_{\mathbf{C}} N_{h} \leq m, \quad \operatorname{codim}_{\mathbf{C}} N_{a} \leq m \text {. }
$$

Observe now that, if $x \in N_{h} \cap N_{a}$, then for every $w \in W, b(x, w)=h(x, w)+$ $a(x, w)=0$, and so $N_{h} \cap N_{a} \subset N$. By (1.4), if $x \in N_{h} \cap N_{a}$, then $s \pi x=x$, and so

$$
(\pi b)(\pi x, \pi x)=h(x, x)+a(x, x), \quad x \in N_{h} \cap N_{a} .
$$

Let $P: \mathbf{C}^{k+n} \rightarrow \mathbf{C}^{k}$ denote the standard projection map. By (1.8), $\operatorname{dim}_{\mathbf{C}} \pi\left(N_{h} \cap N_{a}\right)=\operatorname{dim}_{\mathbf{C}}\left(N_{h} \cap N_{a}\right) \geq k$ (note $n \geq m$ ), and so either 
$\pi\left(N_{h} \cap N_{a}\right) \cap\{0\} \times \mathbf{C}^{n}$ contains a nonzero vector $0 \oplus \xi_{1}$ or $\pi\left(N_{h} \cap N_{a}\right) \cap\{0\} \times \mathbf{C}^{n}=$ (0) and $P\left(\pi\left(N_{h} \cap N_{a}\right)\right)=\mathbf{C}^{k}$.

In the first case, applying (1.9) to $x=s\left(0 \oplus \xi_{1}\right)$ and $x=s\left(0 \oplus J \xi_{1}\right)$, one obtains $(\pi b)\left(0 \oplus \xi_{1}, 0 \oplus \xi_{1}\right)=0,(\pi b)\left(0 \oplus J \xi_{1}, 0 \oplus J \xi_{1}\right)=0$, which means that the Hermitian part of $\pi b$, which is equal to $H_{v}$ by Remark 1.7, vanishes in the direction of $0 \oplus \xi_{1} \in \mathbf{C}^{k} \oplus \mathbf{C}^{n}$, as required.

In the second case, the subspace $\pi\left(N_{h} \cap N_{a}\right)$ is equal to the graph of a C-linear map $S_{0}: \mathbf{C}^{k} \rightarrow \mathbf{C}^{n}$. Since $s \mid \pi\left(N_{h} \cap N_{a}\right)$ is the inverse of a bijective Clinear map $\pi \mid N_{h} \cap N_{a}$ (note that $N_{h} \cap N_{a}$ is a complex subspace), $s \mid \pi\left(N_{h} \cap N_{a}\right.$ ) is C-linear and of the form $s\left(z \oplus S_{0} z\right)=z \oplus S_{0} z \oplus S_{1} z, z \in \mathbf{C}^{k}$, where $S_{1}: \mathbf{C}^{k} \rightarrow \mathbf{C}^{m}$ is C-linear. Let $S z=S_{0} z \oplus S_{1} z$. Then $(\pi b)\left(z \oplus S_{0} z, z \oplus S_{0} z\right)=$ $b\left(s\left(z \oplus S_{0} z, z \oplus S_{0} z\right)\right)=h(z \oplus S z, z \oplus S z)+a(z \oplus S z, z \oplus S z)$. Since the complex Hessian $H_{v}$ of $v$ at $\left(z_{0}, \xi_{0}\right)$ is equal to the Hermitian part of $\frac{1}{2} \pi b$ (see Remark 1.7) and since $z \rightarrow a(z \oplus S z, z \oplus S z)$ is anti-Hermitian, we obtain $H_{v}\left(z \oplus S_{0} z, z \oplus S_{0} z\right)=h(z \oplus S z, z \oplus S z)=H_{u}(z \oplus S z, z \oplus S z)$. Q.E.D.

\section{DUALITY FOR HARMONIC INTERPOLATION}

In this section we give a new, direct proof of the duality theorem for the harmonic interpolation method, which has already been obtained as a special case of a more general duality result for a class of complex interpolation methods in [17, Theorem 6.1, $\S 8.2(\mathrm{~b})$ ]; see also [19, Theorem 2.9]. The present proof is based on a minimum principle for functions of the class $P_{\text {subh }}$ (Lemma 2.1 below), which, as in [17, Theorem 5.1], was inspired by a minimum principle for plurisubharmonic functions due to Kiselman [9, Theorem 2.2]. For the properties of the class $P_{\text {subh }}$, the reader is referred to [19, Introduction and §1]. Recall that a function $v(z, \xi),(z, \xi) \in U \subset \mathbf{C}^{k} \times \mathbf{C}^{n}$, belongs to the dual class $P_{\text {subh }}^{d}(U)$ if for every function $f(z, \xi)$ of class $P_{\text {subh }}(V)$ the sum $v+f$ has local maximum property on $U \cap V$. (See [16, Definition 1.1] for a general definition of the dual class of functions.)

Lemma 2.1. Let $u(z, \xi, w)$ be a function of class $P_{\text {subh }}\left(G \times \mathbf{C}^{n+m}\right)$, where $G$ is open in $\mathbf{C}^{k}$. Assume that $m \leq n$ and that for every $(z, \xi) \in G \times \mathbf{C}^{n}$ the slice function $w \rightarrow u(z, \xi, w)$ is convex. Then the function $v(z, \xi)=$ $\inf \left\{u(z, \xi, w): w \in \mathbf{C}^{k}\right\}$ belongs to $P_{\text {subh }}^{d}\left(G \times \mathbf{C}^{n}\right)$.

Proof. We first prove the claim when $u$ is $\mathbf{C}^{(2)}$-smooth and the slice functions $w \rightarrow u(z, \xi, w),(z, \xi) \in G \times \mathbf{C}^{n}$, are strongly convex. We use the following assertion. (Its proof is practically identical to that of Assertion 2, given below, and is omitted.)

Assertion 1. If $f \in P_{\text {subh }}(V), V \subset \mathbf{C}^{k} \times \mathbf{C}^{n}$, then there exist functions $f_{s} \in\left(P_{\text {subh }} \cap \mathbf{C}^{\infty}\right)\left(V_{s}\right), s=1,2, \ldots$, where $V_{s}=\left\{x \in V: \operatorname{dist}\left(x, V_{s}\right)>1 / s\right\}$, such that for every compact $K \subset V$

$$
f_{s}(z, \xi) \searrow f(z, \xi), \quad(z, \xi) \in K, s \geq N(K) .
$$


Suppose that for a compact $K \subset U \cap V$ and $f_{s}$ as above,

$$
\max \left(v+f_{s}\right)\left|K>\max \left(v+f_{s}\right)\right| \partial K .
$$

By [13, Lemma 4.5], there are $\varepsilon>0$, a point $\left(z_{0}, \xi_{0}\right) \in \operatorname{Int} K$, and an $R$-affine function $l: \mathbf{C}^{k+n} \rightarrow R$ such that

$$
\left(v+f_{s}+l\right)\left(z_{0}, \xi_{0}\right)=0 \geq-\varepsilon\left(\left|z-z_{0}\right|^{2}+\left|\xi-\xi_{0}\right|^{2}\right), \quad(z, \xi) \in K .
$$

We use Lemma 1.1 to show that this is impossible. This is obvious in case there is a nonzero vector $0 \oplus \xi_{1}$, such that $\operatorname{Hess}^{\mathrm{C}} v\left(z_{0}, \xi_{0}\right)$ vanishes in its direction. (Recall that $v$ is a $\mathbf{C}^{(2)}$-smooth function by Remark 1.7.) Since the slice function $z_{0} \rightarrow f_{s}\left(z_{0}, \xi\right)$ is plurisubharmonic by [19, (1.5)], we conclude that $\operatorname{Hess}^{\mathbf{C}}\left(v+f_{s}+l\right)$, at $\left(z_{0}, \xi_{0}\right)$, is positive semidefinite in the direction of $0 \oplus \xi_{1}$, which contradicts (2.3).

In the remaining case there exist C-linear maps $S_{0}$ and $S$ as in Lemma 1.1; in particular, (1.1) holds. By the definition of $P_{\text {subh }}$, the function $z \rightarrow u(z \oplus S z)$ is subharmonic, and so the form $z \rightarrow H_{u}(z \oplus S z, z \oplus S z)$ has nonnegative trace. By (1.1), the form $z \rightarrow H_{v}\left(z \oplus S_{0} z, z \oplus S_{0} z\right)$ has nonnegative trace (recall that $H_{v}=\operatorname{Hess}^{\mathbf{C}} v\left(z_{0}, \xi_{0}\right)$ ). Since $f_{s} \in \mathbf{C}^{(2)} \cap P_{\text {subh }}$, the form $z \rightarrow$ $H_{f_{s}}\left(z \oplus S_{0} z, z \oplus S_{0} z\right)$ also has nonnegative trace (where $\left.H_{f_{s}}=\operatorname{Hess}^{\mathbf{C}} f_{s}\left(z_{0}, \xi_{0}\right)\right)$. Consequently, $z \rightarrow H_{v+f_{s}+l}\left(z \oplus S_{0} z, z \oplus S_{0} z\right)$ has nonnegative trace, which contradicts (2.3) and (2.2). Consequently, $\max \left(v+f_{s}\right)\left|K \leq \max \left(v+f_{s}\right)\right| \partial K$, and by $(2.1)$,

$$
\max (v+f)|K \leq \max (v+f)| \partial K,
$$

and so $v \in P_{\text {subh }}^{d}\left(G \times \mathbf{C}^{n}\right)$, when $u$ is smooth and strongly convex in $w$. To handle the case of general $u$, we need the next assertion.

Assertion 2. Fix $\delta>0$ and denote $u^{\delta}(z, \xi, w)=\max (u(z, \xi, w),-1 / \delta)+$ $\delta|w|^{2}$ and $v^{\delta}(z, \xi)=\inf \left\{u^{\delta}(z, \xi, w): w \in \mathbf{C}^{m}\right\},(z, \xi, w) \in G \times \mathbf{C}^{n} \times \mathbf{C}^{m}$. Then there exist functions $u_{s} \in\left(\mathbf{C}^{\infty} \cap P_{\text {subh }}\right)\left(G_{s} \times \mathbf{C}^{n} \times \mathbf{C}^{m}\right), s=1,2, \ldots$, where $G_{s}=\{x \in G: \operatorname{dist}(x, G)>1 / s\}$, which are strongly convex in $w$ and such that for every compact $L \subset G \times \mathbf{C}^{n}$

$$
u_{s}(z, \xi, w) \searrow u^{\delta}(z, \xi, w), \quad(z, \xi, w) \in L \times \mathbf{C}^{m}, s>n_{0}(L) .
$$

Assuming this, apply (2.4) to $v_{s}(z, \xi)=\inf \left\{u_{s}(z, \xi, w): w \in \mathbf{C}^{m}\right\}$ and obtain

$$
\max \left(v_{s}+f\right)\left|L \leq \max \left(v_{s}+f\right)\right| \partial L .
$$

By the properties of $u_{s}$ and the special construction of $u^{\delta}$, we get $v_{s}(z, \xi) \backslash$ $u^{\delta}(z, \xi),(z, \xi) \in L$, for $s$ large enough, and so by $(2.5), \max \left(v^{\delta}+f\right) \mid L \leq$ $\max \left(v^{\delta}+f\right) \mid \partial L$. It is also clear that $v^{\delta}(z, \xi) \searrow v(z, \xi),(z, \xi) \in G \times \mathbf{C}^{n}$, and so $\max (v+f)|L \leq \max (v+f)| \partial L$, as required. 
As for Assertion 2, the special form of the functions $u^{\delta}, \delta>0$, is not used in the proof (of the assertion), only the fact that each of them is bounded from below.

Let $K_{s}, s=1,2, \ldots$, be the standard, radically symmetric smooth nonnegative convolution kernels on $\mathbf{C}^{k+n+m}$ with decreasing supports, constructed as in Hayman and Kennedy [7, Theorem 3.8]. Seeing that function $u^{\delta}$ is, in particular, subharmonic in the usual sense on $G \times \mathbf{C}^{n} \times \mathbf{C}^{m}[19,(1.6)]$, we get by [7, Theorem 3.8] that $\left(u^{\delta} * K_{s}\right)(z, \xi, w) \searrow u^{\delta}(z, \xi, w)$, for $s \geq N$, if $\operatorname{dist}(z, \partial G)>1 / N$. Since the class $F$ of all functions $u(z, \xi, w)$, which are of class $P_{\text {subh }}$ and convex in $w$, is closed with respect to translations, addition (i.e., $F+F \subset F$ ), and multiplication by nonnegative constants, we conclude that $u^{\delta} * K_{s} \in F\left(G_{s} \times \mathbf{C}^{n+m}\right)$, and so functions $\left(u^{\delta} * K_{s}\right)(z, \xi, w)+a+b|w|^{2}$ belong to $F\left(G_{s} \times \mathbf{C}^{n+m}\right)$.

It is now easy to see that the functions $u_{s}(z, \xi, w)=\left(u^{\delta} * K_{s}\right)(z, \xi, w)+$ $1 / s+(1 / s)|w|^{2}$ satisfy all the requirements of Assertion 2. Q.E.D.

Corollary 2.2. Let $F \in P_{\text {subh }}\left(G \times \mathbf{C}^{n}\right)$, where $G \subset \mathbf{C}^{k}$ is open. Assume that for every $z$, the function $f_{z}(w)=f(z, w)$ is convex on $\mathbf{C}^{n}$. Then the function

$$
g(z, \xi)=\inf \left\{f(z, w)-\operatorname{Re}[\xi, w]: w \in \mathbf{C}^{n}\right\}
$$

is of class $P_{\text {subh }}^{d}\left(G \times \mathbf{C}^{n}\right)$ (the dual class to $\left.P_{\text {subh }}\right)$, where $[\xi, w]=\xi_{1} w_{1}+\cdots+$ $\xi_{n} w_{n}$.

Remark. The function $\xi \rightarrow g(z, \xi)$ is equal to $-\left(f_{z}\right)^{*}$, where $\left(f_{z}\right)^{*}$ denotes the Fenchel conjugate function to $f_{z}$; see Day [6, Chapter VII, §2].

Proof. Observe that the function $u(z, \xi, w)=f(z, \xi)-\operatorname{Re}[\xi, w]$ belongs to $P_{\text {subh }}\left(G \times \mathbf{C}^{2 n}\right)$, by $[19,(1.1)$ and (1.2)]. (Note that the function $(z, \xi, w) \rightarrow$ $\operatorname{Re}[\xi, w]$ is plurisubharmonic and so of class $\left.P_{\text {subh }}\right)$. Since

$$
g(z, \xi)=\inf \left\{u(z, \xi, w): w \in \mathbf{C}^{n}\right\},
$$

Lemma 2.1 yields the conclusion. Q.E.D.

Recall that $\left\{\mathbf{C}^{n}, p_{z}\right\}_{z \in G}$ is called a harmonic subinterpolation family if the function $(z, w) \rightarrow \log p_{z}(w)$ is of class $P_{\text {subh }}\left(G \times \mathbf{C}^{n}\right)$, and that $\left\{\mathbf{C}^{n}, p_{z}\right\}_{z \in G}$ is a harmonic superinterpolation family if the multifunction $z \rightarrow W(z): G \rightarrow$ $w^{\mathbf{C}^{n}}$, where $W(z)=\left\{w \in \mathbf{C}^{n}: p_{z}(w) \leq 1\right\}$, is harmonic. By Characterization $0.5,\left\{\mathbf{C}^{n}, p_{z}\right\}_{z \in G}$ is an interpolation family if and only if it is both a subinterpolation and a superinterpolation family. (See $[17, \S \S 3,4]$ and $[19, \S 2]$ for background and further properties of these notions.)

Theorem 2.3. If the normed spaces $\left\{\mathbf{C}^{n}, p_{z}\right\}_{z \in G}$ form a harmonic subinterpolation family, then their dual spaces $\left\{\mathbf{C}^{n}, q_{z}\right\}_{z \in G}$ form a harmonic superinterpolation family.

Proof. We have to show that $z \rightarrow V(z)$ if a harmonic multifunction, where $V(z)=\left\{w \in \mathbf{C}^{n}: q_{z}(w) \leq 1\right\}$ and $q_{z}(\xi)=\sup \left\{|\langle\xi, w\rangle|: p_{z}(w) \leq 1\right\}$. 
One easily computes that the Fenchel conjugate of the norm $p_{z}(\cdot)$ is

$$
p_{z}^{*}(\xi)= \begin{cases}0 & \text { if } \xi \in W(z), \\ +\infty & \text { if } \xi \notin W(z)\end{cases}
$$

(see also Day [6, Chapter VII, $\S 2(15) \mathrm{h}]$ ); that is, $g \mid X=0$ and $g \mid G \times \mathbf{C}^{n} \backslash X=$ $-\infty$, if $X=\operatorname{gr}(V)$, and $g(z, \xi)=-p_{z}^{*}(\xi)$. By Lemma 2.2, $g \in P_{\text {subh }}^{d}$, and so, if $B$ and $u(z, w)$ are as in Definition 0.2, then

$$
\max u|X \cap B=\max (u+g)| B \leq \max (u+g)|\partial B=\max u|(\partial B) \cap X,
$$

which shows that $V(\cdot)$ is a harmonic multifunction. Q.E.D.

Proof of Theorem 0.7. In view of the last theorem and comments preceding it, we have only to show that, if $\left\{\mathbf{C}^{n}, p_{z}(\cdot)\right\}_{z \in G}$ is a harmonic superinterpolation family, then its dual $\left\{\mathbf{C}^{n}, q_{z}(\cdot)\right\}$ is a harmonic subinterpolation family. (The argument we are about to sketch is practically the same as the more general one in [17, Corollary 2.4]).

In view of Definition 0.1 , we have to prove that whenever $\xi=f(z)$ is an analytic mapping, the function $v(z)=q_{z}(f(z))$ is subharmonic. Clearly, this is equivalent to showing that for every harmonic function $h(z)$ and every compact set $F \subset \operatorname{dom}(v) \cap \operatorname{dom}(h), \max (v+h)|F \leq \max (v+h)| \partial F$. Now the function $u(z, w)=|\langle w, f(z)\rangle|+h(z)$ is of class $P_{\text {subh }}$ and so, seeing that the multifunction $z \rightarrow W(z)$, where $W(z)=\left\{w \in \mathbf{C}^{n}: p_{z}(w) \leq 1\right\}$, is harmonic (we have superinterpolation family), we get, by Definition $0.2, \max (v+h) \mid F=$ $\max u\left|\operatorname{gr}(W) \cap\left(F \times \mathbf{C}^{n}\right) \leq \max u\right| \operatorname{gr}(W) \cap\left(\partial F \times \mathbf{C}^{n}\right)=\max (v+h) \mid \partial F$. Q.E.D.

Proof of Characterization 0.6. In view of the duality properties of subinterpolation and superinterpolation families, it is now clear that $\left\{\mathbf{C}^{n}, p_{z}\right\}_{z \in G}$ is a harmonic interpolation family if and only if it is a subinterpolation family and the dual spaces $\left\{\mathbf{C}^{n}, q_{z}\right\}_{z \in G}$ form a subinterpolation family. This observation reduces Characterization 0.6 to the next assertion.

Assertion. The function $(z, w) \rightarrow \log p_{z}(w)$ lies in $P_{\text {subh }}\left(G \times \mathbf{C}^{n}\right)$ if and only if the function $(z, w) \rightarrow \frac{1}{2} p_{z}(w)^{2}$ is of class $P_{\text {subh }}$.

Denote $f(z, w)=\frac{1}{2} p_{z}(w)^{2}$. Since $f(z, w)=\exp \left(\frac{1}{2} \log p_{z}(w)\right)$, necessity is clear. Conversely, since $f$ is homogeneous, of order 2 in $w$, the function $(z, w) \rightarrow\left|e^{h(z)}\right| f(z, w)=f\left(z, e^{(1 / 2) h(z)} w\right)$ is of class $P_{\text {subh }}$, which easily yields that $\log f$ lies in $P_{\text {subh }}$; see [18, Lemma 5.3]. Q.E.D.

The reader who does not want to consult [17] can skip Remark 2.4 and Lemma 2.5.

Remark 2.4. Obvious modifications of the above arguments would yield easily a new proof of the following result (which is a special class of [17, Theorem 6.1]): if $Q$ is a pseudoconvex class of functions on $\mathbf{C}^{k}$ satisfying conditions [17, (1.1)-(1.12)] and such that $Q+Q \subset Q$, then $\left\{\mathbf{C}^{n}, p_{z}\right\}_{z \in G}$ is a $P_{Q}^{c o}$-interpolation 
family of normed spaces if and only if the dual spaces $\left\{\mathbf{C}^{n}, q_{z}\right\}_{z \in G}$ form a $P_{Q^{d-}}^{c o}$ interpolation family (see $[17, \S 2]$ for notation and definition). The key element of proof is the following generalization of Lemma 2.1.

Lemma 2.5. If $Q$ is as in Remark 2.4 (in particular, $Q+Q \subset Q, u \in$ $\left.P_{Q}^{p s h}\left(G \times \mathbf{C}^{n+m}\right)\right)$ and is convex in the direction $\{0\} \times\{0\} \times \mathbf{C}^{m}$, then $v \in\left(P_{Q^{d}}^{p s h}\right)^{d}$, where $v(z, \xi)=\inf \left\{u(z, \xi, w): w \in \mathbf{C}^{m}\right\},(z, \xi) \in G \times \mathbf{C}^{n}$.

The proof proceeds along the lines of the proof of Lemma 2.1. The property $Q+Q \subset Q$ is used only to obtain analogs of Assertions 1 and 2, namely in the statement that $u^{\delta} * K_{s} \in P_{Q}$. We omit further details.

\section{Complex Hessian and duality}

Lemma 3.1. Let $Z, W$ be finite-dimensional complex vector spaces. Let

$$
\frac{1}{2}\left\{\left\langle B_{12} w, z^{\prime}\right\rangle+\left\langle B_{21} z, w^{\prime}\right\rangle+\left\langle B_{22} w, w^{\prime}\right\rangle\right\}
$$

be a real symmetric bilinear form (where $B_{i j}$ are as in Proposition 1.6), with Hermitian part equal to

$$
\left\langle H_{12} w, z^{\prime}\right\rangle+\left\langle H_{21} z, w^{\prime}\right\rangle+\left\langle H_{22} w, w^{\prime}\right\rangle
$$

where $H_{12}^{*}=H_{21}, H_{22}^{*}=H_{22}$. Assume that $B_{22}$ is positive definite. Let

$$
\left\langle\widetilde{H}_{0}, z, z^{\prime}\right\rangle+\left\langle\widetilde{H}_{12} \xi, z^{\prime}\right\rangle+\left\langle\widetilde{H}_{21} z, \xi^{\prime}\right\rangle+\left\langle\widetilde{H}_{22} \xi, \xi^{\prime}\right\rangle
$$

denote the Hermitian part of the form

$$
\frac{1}{2}\left\{\left\langle-B_{12} B_{22}^{-1} B_{21} z, z^{\prime}\right\rangle+\left\langle B_{12} B_{22}^{-1} \xi, z^{\prime}\right\rangle+\left\langle B_{22}^{-1} B_{21} z, \xi\right\rangle+\left\langle-B_{22}^{-1} \xi, \xi^{\prime}\right\rangle\right\} .
$$

Then $\widetilde{H}_{0}-\widetilde{H}_{12} \widetilde{H}_{22}^{-1} \widetilde{H}_{21}=-H_{12} H_{22}^{-1} H_{21}$.

This admittedly technical formulation is motivated by the convenience of application in $\S 4$. (In particular, terms $B_{11}, H_{11}$ are omitted because they have no local sense in the context of $\S 4$.) Before proving the lemma, we give some comments which should explain its meaning.

Note first that the pairing $\langle$,$\rangle is real valued, and so forms (3.2), (3.3) are$ actually the real parts of usual, complex-valued Hermitian forms. Complex structure is given by an operator $J$, e.g., $J: Z \rightarrow Z$ with $J^{2}=-I$ and $H_{i j}$ are $J$-antilinear operators; e.g., operator $\frac{1}{2} B_{12}: W \rightarrow Z^{*}$ splits uniquely into $\frac{1}{2} B_{12}=H_{12}+A_{12}$, where $J H_{12}=-H_{12} J, J A_{12}=A_{12} J$. (The pairing $\langle$, satisfies $\langle J x, J y\rangle=-\langle x, y\rangle$.

In view of $\S 1$, if $h(z \oplus w, z \oplus w)$ is a Hermitian form with the four blocks equal respectively to $H_{11}, H_{12}, H_{21}, H_{22}$, then the term $H_{11}-H_{12} H_{22}^{-1} H_{21}=$ $\pi h$, the projection of $h$ onto $Z$ under the map $\pi: Z \oplus W \rightarrow Z$. The next proposition is a special case of the results from [18, Lemmas 4.11(ii) and 4.12(ii)], reformulated in the language of projections. 
Proposition 3.2. Let $f(z, w)$ be a $\mathbf{C}^{(2)}$-smooth on $U \subset \mathbf{C}^{k+n}, U$ open. Then

(i) if $f$ is strictly plurisubharmonic in $w$, then $f \in P_{\text {subh }}(U)$ if and only if $\operatorname{tr} \pi\left(\operatorname{Hess}^{\mathbf{C}} f(z, w)\right) \geq 0$ in $U$;

(ii) if $f$ is strictly plurisuperharmonic in $w$, then $f \in\left(P_{\text {subh }}\right)^{d}(U)$, if and only if $\operatorname{tr} \pi\left(\operatorname{Hess}^{\mathrm{C}} f(z, w)\right) \geq 0$ in $U$.

The next proposition explains the role of Lemma 3.1 in the smooth case.

Proposition 3.3. Let $f(z, w)$ be a $\mathbf{C}^{(2)}$-function on $G \times \mathbf{C}^{n}, G \subset \mathbf{C}^{k}$. Assume that $f$ is strongly convex in $w$ and let $g(z, \xi)=\inf \{f(z, w)-\operatorname{Re}[\xi, w]: w \in$ $\left.\mathrm{C}^{n}\right\}$. Denote by $w(\xi)$ the unique point where the infimum is attained. Then the projections, onto $\mathbf{C}^{k}$, of the forms Hess ${ }^{\mathbf{C}} f\left(z_{0}, w_{0}\right)$ and Hess ${ }^{\mathbf{C}} g\left(z_{0}, \boldsymbol{\xi}_{0}\right)$, where $w_{0}=w\left(\xi_{0}\right)$, under the maps $\pi(z, w)=z$ and $\tilde{\pi}(z, \xi)=z$ are equal.

Proof (sketch). If the real Hessian of $f$ at $\left(z_{0}, w_{0}\right)$ has block components $B_{11}$, $B_{12}, B_{21}, B_{22}$ as in (1.6), one can compute, using the implicit function theorem (Remark 1.5 and Proposition 1.6), that $\frac{1}{2} \operatorname{Hess}^{R} g\left(z_{0}, \xi_{0}\right)=\frac{1}{2}\left\langle B_{11} z, z^{\prime}\right\rangle+$ terms (3.4), and so its Hermitian part differs from (3.3) by the term $\left\langle H_{11} z, z^{\prime}\right\rangle$. With these observations, Lemma 3.1 now implies immediately that both $\pi\left(\frac{1}{2}\right.$ Hess $\left.^{\mathbf{C}} f\left(z_{0}, w_{0}\right)\right)$ and $\tilde{\pi}\left(\frac{1}{2}\right.$ Hess $\left.^{\mathbf{C}} g\left(z_{0}, \xi_{0}\right)\right)$ are equal to $H_{11}-H_{12} H_{22}^{-1} H_{21}$. Q.E.D.

Remark 3.4. Combining Propositions 3.2 and 3.3, we obtain a new proof of Corollary 2.2

Proof of Lemma 3.1. Represent $\frac{1}{2} B_{i j}=H_{i j}+A_{j}$, where $-H_{i j} J=J H_{i j}, A_{i j} J=$ $J A_{i j}$. Note that $H_{22}$ is positive definite as the Hermitian part of the positive definite $\frac{1}{2} B_{22}$. (See e.g., the proof of the assertion below.) Represent, further, $2 B_{22}^{-1}=S+N$, where $-S J=J S, N J=J N$. Then, by direct computation,

$$
\begin{gathered}
\tilde{H}_{0}=-H_{12} S H_{21}-A_{12} S A_{21}-A_{12} N H_{21}-H_{12} N A_{21}, \\
\widetilde{H}_{12}=\frac{1}{2} A_{12} S+\frac{1}{2} H_{12} N, \quad \widetilde{H}_{21}=\frac{1}{2} S A_{21}+\frac{1}{2} N H_{21}, \\
\tilde{H}_{22}=-\frac{1}{4} S .
\end{gathered}
$$

Further computation yields

$$
\widetilde{H}_{0}-\widetilde{H}_{12} \widetilde{H}_{22}^{-1} \widetilde{H}_{21}=-H_{12}\left(S-N S^{-1} N\right) H_{21} .
$$

To evaluate this, identify $S$ and $N$. Let $\psi=H_{22}^{-1} A_{22}$. Then

$$
2 B_{22}^{-1}=\left(H_{22}\left(I+H_{22}^{-1} A_{22}\right)\right)^{-1}=(I+\psi)^{-1} H_{22}^{-1} \text {. }
$$

Assertion. Spectrai radius of $\psi$ is smaller than one.

Assuming the assertion, we get

$$
2 B_{22}^{-1}=\left(\sum_{r=0}^{\infty}(-1)^{r} \psi^{r}\right) H_{22}^{-1}=\left(\sum_{r=0}^{\infty} \psi^{2 r}\right) H_{22}^{-1}-\psi\left(\sum_{r=0}^{\infty} \psi^{2 r}\right) H_{22}^{-1} .
$$


Since $\psi J=-J \psi$, we conclude that $S=\left(I-\psi^{2}\right)^{-1}$ and $N=\psi\left(I-\psi^{2}\right)^{-1} H_{22}^{-1}$. Hence,

$$
\begin{aligned}
S-N S^{-1} N= & \left(I-\psi^{2}\right)^{-1} H_{22}^{-1} \\
& -(-1) \psi\left(I-\psi^{2}\right)^{-1} H_{22}^{-1} H_{22}\left(I-\psi^{2}\right)(-1) \psi\left(I-\psi^{2}\right)^{-1} H_{22}^{-1} \\
= & \left(I-\psi^{2}\right)^{-1} H_{22}^{-1}-\psi^{2}\left(I-\psi^{2}\right)^{-1} H_{22}^{-1}=H_{22}^{-1} .
\end{aligned}
$$

Substituting this into (3.8), we get $\widetilde{H}_{0}-\widetilde{H}_{12} \widetilde{H}_{22}^{-1} \widetilde{H}_{21}=-H_{12} H_{22}^{-1} H_{21}$, as required.

It remains to check the assertion. Since $\left\langle B_{22} w, w\right\rangle>0$ and $\left\langle B_{22}(J w)\right.$, $J w\rangle>0$ for $w \neq 0, w \in W$, we get

$$
\begin{aligned}
0< & \left\langle\frac{1}{2} B_{22}\left(w_{1}-w_{2}\right),\left(w_{1}-w_{2}\right)\right\rangle+\left\langle\frac{1}{2} B_{22}\left(J w_{1}+J w_{2}\right), J w_{1}+J w_{2}\right\rangle \\
= & \left\langle H_{22}\left(w_{1}-w_{2}\right), w_{1}-w_{2}\right\rangle+\left\langle A_{22}\left(w_{1}-w_{2}\right), w_{1}-w_{2}\right\rangle \\
& +\left\langle H_{22}\left(w_{1}+w_{2}\right), w_{1}+w_{2}\right\rangle-\left\langle A_{22}\left(w_{1}+w_{2}\right), w_{1}+w_{2}\right\rangle \\
= & 2\left\langle H_{22} w_{1}, w_{1}\right\rangle+2\left\langle H_{22} w_{2}, w_{2}\right\rangle-2\left\langle A_{22} w_{1}, w_{2}\right\rangle-2\left\langle A_{22} w_{2}, w_{1}\right\rangle .
\end{aligned}
$$

unless $w_{1}+w_{2}=0=w_{1}-w_{2}$, i.e., unless $w_{1}=w_{2}=0$. Since $A_{22}^{T}=A_{22}$ and $A_{22} \psi=H_{22}$, we conclude that $\left|\left\langle H_{22} \psi w_{1}, w_{2}\right\rangle\right|<1$ whenever $\left\langle H_{22} w_{1}, w_{1}\right\rangle=$ $1=\left\langle H_{22} w_{2}, w_{2}\right\rangle$. This means that the operator norm of $\psi$, relative to the norm $w \rightarrow\left\langle H_{22} w, w\right\rangle^{1 / 2}$ on $W$, is smaller than one, and so is its spectral radius. Q.E.D.

\section{ChaRACTERIZATION OF HARMONIC INTERPOlation FAMILIES IN TERMS OF SECOND-ORDER DERIVATIVES}

We first characterize functions of class $P_{\text {subh }}$ in terms of their complex Hessian (Lemma 4.4) and then compute real and complex Hessians of the partial Fenchel conjugate (Lemma 4.6). From these two facts, Theorems 0.10 and 0.11 will follow quickly.

Notation 4.1. If $f(x, y)$ is an $L_{\text {loc }}^{1}$ function defined on a subset of $R^{K} \times$ $R^{N}$, we denote (in analogy with Notation 0.9) by $B_{11} f, B_{12} f, B_{21} f, B_{22} f$ the blocks of the distributional real Hessian of $f(x, y)$, namely the matrixvalued distributions $\left(\partial^{2} f / \partial x_{i} \partial x_{j}\right)_{i j},\left(\partial^{2} f / \partial x_{i} \partial y_{q}\right)_{i q},\left(\partial^{2} f / \partial y_{p} \partial x_{j}\right)_{p j}$, and $\left(\partial^{2} f / \partial y_{p} \partial y_{q}\right)_{p q}$ (of sizes $K \times K, K \times N, N \times K$, and $N \times N$, respectively).

Proposition 4.2. Let $f: \Omega \times R^{N} \rightarrow R$, with $\Omega \subset R^{K}$ open, be continuous and such that $\lim _{|y| \rightarrow \infty} f(x, y) /|y|=+\infty$, for $x \in \Omega$. Assume further that $f(x, y)$ is locally uniformly smooth and locally uniformly convex in $y$, that is,

$$
\begin{aligned}
2 f(x, y)+2 \varepsilon|h|^{2} & \leq f(x, y+h)+f(x, y-h) \\
& \leq 2 f(x, y)+2 K|h|^{2}, \quad|h| \leq \eta,
\end{aligned}
$$


with $K<+\infty$ and $\varepsilon, \eta>0$, uniformly on compact subsets of $\Omega \times R^{N}$. Then

(i) the distribution $B_{22} f$ is represented by an $L_{\mathrm{loc}}^{\infty}$, matrix-valued function, with $B_{22} f(x, y)$ positive definite for a.a. $(x, y) \in \Omega \times R^{N}$, and $\left(B_{22} f\right)^{-1} \in$ $L_{\mathrm{loc}}^{\infty}$;

(ii) the map $y \rightarrow G_{x}(y): R^{N} \rightarrow R^{N}$, where

$$
G_{x}(y)=\left(\partial f / \partial y_{1}, \ldots, \partial f / \partial y_{n}\right), \quad(x, y) \in \Omega \times R^{N},
$$

is locally bi-Lipschitz, with Lipschitz constants locally uniformly bounded in $(x, y)$;

(iii) the map

$$
G: \Omega \times R^{N} \rightarrow \Omega \times R^{N}, \quad \text { where } G(x, y)=\left(x, G_{x}(y)\right),
$$

is a homeomorphism onto.

Proof (sketch). As is well known, (4.1) is equivalent to both functions $y \rightarrow$ $K|y|^{2}-f(x, y)$ and $y \rightarrow f(x, y)-\varepsilon|y|^{2}$ being convex for $(x, y)$ near $\left(x_{0}, y_{0}\right)$. Since a function in $R^{N}$ is convex if and only if its real Hessian is a matrixvalued distribution with positive semidefinite values, (i) holds; in particular,

$$
\varepsilon|h|^{2} \leq\left(B_{22}(x, y) h, h\right) \leq K|h|^{2}
$$

for $(x, y)$ near $\left(x_{0}, y_{0}\right)$. These inequalities imply $\varepsilon\left|y-y_{0}\right| \leq \mid G_{x}(y)-$ $G_{x}\left(y_{0}\right)|\leq K| y-y_{0} \mid$ for $y$ near $y_{0}$. Finally, (iii) is geometrically obvious. Q.E.D.

Remark 4.3. Observe that, if $\left\{\mathbf{C}^{n},\|\cdot\|_{z}\right\}_{z \in \bar{G}}$ is a harmonic interpolation family satisfying assumptions of Property 0.8 , then the function $f(z, w)=\frac{1}{2}\|w\|_{z}^{2}$ satisfies assumptions of Proposition 4.2. In particular, (4.4) holds, and so

$$
\begin{gathered}
H_{22} f \in L_{\mathrm{loc}}^{\infty}\left(G \times \mathbf{C}^{n}\right), \\
\left(H_{22} f\right)^{-1} \in L_{\mathrm{loc}}^{\infty}\left(G \times \mathbf{C}^{n}\right),
\end{gathered}
$$

This motivates the setup of the next lemma.

Lemma 4.4. Let $u(z, w)$ be a continuous function on $U \subset \mathbf{C}^{k} \times \mathbf{C}^{n}$ satisfying condition (4.5). Then

(a) $u \in P_{\text {subh }}(U)$ if and only if, for every (constant) $n \times k$ complex matrix $S$, the distribution

$$
\operatorname{tr}\left(H_{11} u+\left(H_{12} u\right) S+S^{*}\left(H_{21} u\right)+S^{*}\left(H_{22} u\right) S\right)
$$

is (represented by) a nonnegative Borel measure on $U$;

(b) if $u \in P_{\text {subh }}(U)$, then the distributions $H_{12} u, H_{21} u, \partial^{2} u / \partial z_{i} \partial w_{p}$, $i=1, \ldots, k, p=1, \ldots, n$, are (represented by) $L_{\mathrm{loc}}^{2}(U)$ functions and the (distributional) "partial Laplacian" $\operatorname{tr}\left(H_{11} u\right)=\sum_{i=1}^{k} \partial^{2} u / \partial \bar{z}_{i} \partial z_{i}$ is a nonnegative Borel measure on $U$; 
(c) if $\partial^{2} u / \partial \bar{z}_{i} \partial w_{j} \in L_{\mathrm{loc}}^{2}(U), i=1, \ldots, k, p=1, \ldots, n$, and $\operatorname{tr}\left(H_{11} u\right)$ is a Borel measure on $U$, then $u \in P_{\text {subh }}(U)$ if and only if

the measure $\operatorname{tr}\left(H_{11} u\right)-\operatorname{tr}\left(H_{12} u\right)(x)\left(H_{22} u\right)(x)^{-1}\left(H_{21} u\right)(x) d m$ is nonnegative.

We will use the following observations (presumably well known) in the proof of Lemma 4.4 .

Remark 4.5. (a) If $u: G \times H \rightarrow R, G \subset R^{k}, H \subset R^{n}$, is a continuous function, then for every $y \in H$, the function $x \rightarrow u(x, y): G \rightarrow R$ is subharmonic if and only if the distribution $\left(\sum_{i=1}^{k} \partial^{2} / \partial x_{i}^{2}\right) u$ is represented by a nonnegative Borel measure $\mu$ on $G \times H$.

(b) If $u(x, y)$ is a bounded, upper-semicontinuous function on $G \times H \subset$ $R^{k} \times R^{n}$, which is subharmonic in $x$, then the partial gradient $\nabla_{x} u=(\partial u / \partial x$, $\ldots, \partial u\left(\partial x_{k}\right)$ is locally square-integrable. Moreover, if $E$ is relatively compact in $G$, then

$$
\sup _{y \in H} \operatorname{ess}\left(\int_{E}\left|\left(\nabla_{x} u\right)(x, y)\right|^{2} d m(x)\right)^{1 / 2} \leq \mathbf{C} e^{\|u\|_{\infty}},
$$

where $\mathrm{C}$ depends only on $G$ and $E$.

(c) If $u(z, w)$ is continuous on $G \times H \subset \mathbf{C}^{k} \times \mathbf{C}^{n}, \nabla_{z} u \in L_{\text {loc }}^{2}$, and $\partial^{2} u / \partial w_{j} \partial \bar{z}_{i} \in L_{\text {loc }}^{2}$, then $\partial^{2} u / \partial \bar{w}_{j} \partial \bar{z}_{i} \in L_{\text {loc }}^{2}$.

Proof (sketch). (a) is obtained by Fubini-like modification of the standard characterization of subharmonic functions in terms of the distributional Laplacian; see [7, §3.5, Lemma 3.6];

(b) is proved by the identity $\Delta_{x} e^{u}=e^{u} \Delta_{x} u+e^{u}\left|\Delta_{x} u\right|^{2}$; and

(c) is obtained by using the Fourier transform of $u$ (multiplied by a smooth cutoff function) and the fact that $L^{\infty}$ functions are multipliers for the Fourier transforms of $L^{2}$ functions. Q.E.D.

Proof of Lemma 4.4. (a) By Remark 4.5(a), we conclude that $u \in P_{\text {subh }}$ if and only if the distribution

$$
\left(\sum_{i=1}^{k} \frac{\partial^{2}}{\partial z_{i} \partial \bar{z}_{i}}\right)[u(z, w)+S(z)]
$$

is a nonnegative measure on $U$. By standard computations on test functions, distribution (4.9) is equal to the composition of the distribution

$$
\operatorname{tr}\left(H_{11} u+\left(H_{12} u\right) S+S^{*}\left(H_{21} u\right)+S^{*}\left(H_{22} u\right) S\right)
$$

with the affine map $(z, w) \rightarrow(z, w+s(z))$. The latter map being nonsingular, distribution (4.9) is a nonnegative measure if and only if so is distribution (4.10). 
(b) Substituting $S=0$, we get that $\operatorname{tr}\left(H_{11} u\right)=\sum_{i=1}^{k} \partial^{2} u / \partial z_{i} \partial \bar{z}_{i}$ is represented by a nonnegative measure, say $\mu$. Now fix $i, j$ with $1 \leq i \leq k$, $1 \leq j \leq n$, and let $S=\left(a_{p q}\right)$, where $a_{p q}=\alpha \delta_{i p} \delta_{j q}, 1 \leq p \leq k, 1 \leq q \leq n$, with $\alpha \in \mathbf{C}$. Then (4.7) reduces to

$$
\mu+2 \operatorname{Re}\left(\alpha \frac{\partial^{2} u}{\partial \bar{z}_{i} \partial w_{j}}\right)+\alpha^{2} \frac{\partial^{2} u}{\partial \bar{w}_{j} \partial w_{j}} .
$$

This being a Borel measure for every $\alpha \in \mathbf{C}$, the distribution $\partial^{2} u / \partial \bar{z}_{i} \partial w_{j}$ must be represented by a complex Borel measure, say $\mu_{i j}$. (Recall that $\partial^{2} u / \partial \bar{w}_{i} \partial w_{j}$ $\in L^{\infty}$.)

Observe that $\mu_{i j}$ is absolutely continuous with respect to the Lebesgue measure $m$. Indeed, let $F$ be a compact set with $m(F)=0$. The integral of the function $1_{F}$ with respect to the measure (4.11), which is equal to $\mu(F)+$ $2 \operatorname{Re}\left(\alpha \mu_{i j}(F)\right)$, must be nonnegative for every $\alpha \in \mathbf{C}$. Hence, $\mu_{i j}(F)=0$, as required.

Using $L_{\text {loc }}^{1}$ matrix-valued functions $H_{i j}(x), i, j=1,2$, we can interpret (4.10) as

$$
\frac{d \mu}{d m}(x)+\operatorname{tr}\left(\left(H_{12} u\right)(x) S+S^{*}\left(H_{21} u\right)(x)+S^{*}\left(H_{22} u\right)(x) S\right) \geq 0, \quad \text { a.e. } d m .
$$

Assume first that $\left(H_{22} u\right)(x)$ is a nonsingular matrix for a.a. $x$ (and so, positive definite a.e.); (4.12) is equivalent to

$$
\begin{aligned}
0 & \leq\left[\frac{d \mu}{d m}(x)-\operatorname{tr}\left(H_{12} u\right)(x)\left(H_{22} u\right)(x)^{-1}\left(H_{21} u\right)(x)\right]+\operatorname{tr} R(x)^{*} R(x) \\
& =\left(\frac{d \mu}{d m}(x)-\left\|\left(H_{22} u\right)(x)^{1 / 2}\left(H_{21} u\right)(x)\right\|^{2}\right)+\operatorname{tr} R(x)^{*} R(x),
\end{aligned}
$$

where $R(x)=\left(H_{22} u\right)(x)^{-1 / 2}\left(H_{21} u\right)(x)+\left(H_{22} u\right)(x)^{1 / 2} S$, and ||$\cdot \mid \|$ stands for the Hilbert-Schmidt norm of a matrix. Since $S$ is arbitrary, we get $\left\|\left(H_{22} u\right)(x)^{-1 / 2}\left(H_{21} u\right)(x)\right\|^{2} \leq d \mu(x) / d m$, a.e. $d m$. Since $H_{22}(\cdot) \in L_{\text {loc }}^{\infty}$, we conclude that $\left(H_{21} u\right)(x)$ is a square-integrable (matrix-valued) function.

In case not all $\left(H_{22} u\right)(x)$ are nonsingular, let $v(z, w)=u(z, w)+|w|^{2}$. Then, $v \in P_{\text {subh }}(U)$, and $\left(H_{22} v\right)(x)=\left(H_{22} u\right)(x)+\left(\delta_{i j}\right)$ is a nonsingular matrix a.e. $d m$. Since $\left(H_{21} v\right)(x)=\left(H_{21} u\right)(x)$, the previous argument implies that $\left(H_{21} u\right)(\cdot) \in L_{\text {loc }}^{2}$ (as well as $\left.H_{12} u=H_{21} u\right)$.

By Remark 4.5(b), $\partial u / \partial \bar{z}_{i} \in L_{\text {loc }}^{2}$, and since $\partial^{2} u / \partial w_{j} \partial \bar{z}_{i} \in L_{\text {loc }}^{2}$, Remark 4.5(c) implies that $\partial^{2} u / \partial \bar{w}_{j} \partial \bar{z}_{i} \in L_{\text {loc }}^{2}$.

(c) The arguments used above imply also that, under the assumptions of (c), conditions (4.7) and (4.8) are equivalent. We omit further details. Q.E.D.

For the proofs of Theorems 0.10 and 0.11 , we now compute the real and complex Hessians of the partial Fenchel conjugate under weak differentiability assumptions suggested by conclusions of Lemma 4.4 . 
If $F: G_{1} \rightarrow G$ is a continuous map and $\mu_{1}$ is a Radon measure on $G$, we denote by $F_{*} \mu_{1}$ the continuous image of $\mu_{1}$ under $F$, that is, $\left(F_{*} \mu_{1}\right)(B)=$ $\mu_{1}\left(F^{-1}(B)\right.$, for a Borel subset $B \subset G$; see $[8, \S 12.45]$.

Lemma 4.6. (a) Let $f: \Omega \times R^{N} \rightarrow R, \Omega \subset R^{K}$, be as in Proposition 4.2; in particular $B_{22} f,\left(B_{22} f\right)^{-1} \in L_{\mathrm{loc}}^{\infty}$. Assume further that $\nabla_{x} f \in L_{\mathrm{loc}}^{2}, B_{12} f \in L_{\mathrm{loc}}^{2}$ and that $\operatorname{tr} B_{11} f$ is a Radon measure on $\Omega \times R^{N}$. Let

$$
g(x, \eta)=\inf \left\{f(x, y)-\langle\eta, y\rangle: y \in R^{N}\right\}, \quad(x, \eta) \in G \times R^{N},
$$

and let $F=G^{-1}$; see (4.3). Denote $\widetilde{B}_{i j}=B_{i j} g, B_{i j}=B_{i j} f, i, j=1,2$. Then $\widetilde{B}_{22}, \widetilde{B}_{22}^{-1} \in L_{\mathrm{loc}}^{\infty}, \widetilde{B}_{12} \in L_{\mathrm{loc}}^{2}$, and $\operatorname{tr} \widetilde{B}_{11}$ is a Radon measure on $\Omega \times R^{N}$. Furthermore,

$$
\begin{gathered}
\widetilde{B}_{22}(x, \eta)=-B_{22} \circ F(x, \eta)^{-1}, \quad \text { a.e. } \\
\widetilde{B}_{12}=\left(B_{12} \circ F\right)\left(B_{22} \circ F\right)^{-1} \quad \text { a.e., } \quad \widetilde{B}_{21}=\left(B_{21} \circ F\right)\left(B_{22} \circ F\right)^{-1}, \quad \text { a.e., } \\
\operatorname{tr} \widetilde{B}_{11}=\frac{1}{\chi} G_{*}\left(\operatorname{tr} B_{11}\right)-\operatorname{tr}\left(B_{12} B_{22}^{-1} B_{21}\right) \circ F d m,
\end{gathered}
$$

where $m$ is the Lebesgue measure in $\Omega \times R^{N}$ and $\chi=d G_{*}(m) / d m$.

(b) If, in addition, $R^{K}=R^{2 k}=\mathbf{C}^{k}$ and $R^{N}=R^{2 n}=\mathbf{C}^{n}$, denote $H_{i j}=$ $H_{i j} f, \widetilde{H}_{i j}=H_{i j} g, i, j=1,2$; see Notation (0.9). Assume further that $\operatorname{tr} H_{11} \in L_{\text {loc }}^{1}$. Then $\operatorname{tr} \widetilde{H}_{11} \in L_{\mathrm{loc}}^{1}$, and

$$
\begin{aligned}
& \left(\operatorname{tr} \widetilde{H}_{11}-\operatorname{tr} \widetilde{H}_{12} \widetilde{H}_{22}^{-1} \widetilde{H}_{21}\right)(z, \xi) \\
& \quad=\left(\operatorname{tr} H_{11}-\operatorname{tr} H_{12} H_{22}^{-1} H_{21}\right) \cdot F(z, \xi) \text { a.e. } d m .
\end{aligned}
$$

The proof is based on the next three propositions and Lemma 0.12, which play the role of chain rule in our context.

Definition 4.7. Let $F: G_{1} \rightarrow G$ be a homeomorphism, $G, G_{1} \subset R^{n}$. Denote by $m$ and $m_{1}$ the Lebesgue measure on $G$ and $G_{1}$, respectively. We say that $F$ has locally bounded volume distortion if the measures $m$ and $F_{*} m_{1}$ are mutually absolutely continuous and $d m / d\left(F_{*} m_{1}\right), d\left(F_{*} m_{1}\right) / d m \in L_{\mathrm{loc}}^{\infty}$.

Proposition 4.8. Under assumptions of Proposition 4.2, the map $G$ and its inverse (see (4.2), (4.3)) have locally bounded volume distortion.

Lemma 4.9. Let $F: G_{1} \rightarrow G, G_{1}, G \subset R^{n}$, be a homeomorphism with locally bounded volume distortion.

(a) If $u \in L_{\mathrm{loc}}^{p}(G, m)$, then $u \circ F \in L_{\mathrm{loc}}^{p}\left(G_{1}, m_{1}\right), 1 \leq p \leq+\infty$.

(b) If $p \in[1,+\infty]$ and $u_{n} \rightarrow u$ in $L_{\mathrm{loc}}^{p}(G, m)$, then $u_{n} \circ F \rightarrow u \circ F$ in $L_{\mathrm{loc}}^{p}\left(G_{1}, m_{1}\right)$. 
(c) If $p, q \in[1,+\infty],(1 / p)+(1 / q)=1, i \in\{1,2, \ldots, n\}, u \in$ $L_{\mathrm{loc}}^{1}(G, m), \partial u / \partial y_{1}, \ldots, \partial u / \partial y_{n} \in L_{\mathrm{loc}}^{q}(G, m), \partial F_{1} / \partial x_{i}, \ldots, \partial F_{n} / \partial x_{i} \in$ $L_{\mathrm{loc}}^{p c}\left(G_{1}, m_{1}\right)$ (where all the derivatives are in the sense of distributions), then the distributional derivative $\partial(u \circ F) / \partial x_{i}$ is represented by an $L_{\mathrm{loc}}^{1}\left(G_{1}, m\right)$ function, namely

$$
\frac{\partial}{\partial x_{i}}(u \circ F)=\sum_{j=1}^{n}\left(\frac{\partial u}{\partial y_{i}}\right) \circ F \frac{\partial F}{\partial x_{i}} \text { a.e. } d m \text {. }
$$

Lemma 4.10. Let $G, F, \Omega$ be as in Lemma 0.12 and let $Y=\left(Y_{1}, \ldots, Y_{k}\right) \in$ $L_{\mathrm{loc}}^{1}\left(\Omega \times R^{n}\right)$. Assume that $\partial Y / \partial y_{j} \in L_{\mathrm{loc}}^{2}, j=1, \ldots, n$, and that the distribution $\operatorname{div}_{x} Y=\sum_{i=1}^{k} \partial Y_{i} / \partial x_{i}$ is represented by a Radon measure on $\Omega \times R^{n}$. Then $Y \circ F \in L_{\mathrm{loc}}^{1}$ and $\operatorname{div}_{x}(Y \circ F) \in L_{\mathrm{loc}}^{2}$. Furthermore,

$$
\operatorname{div}_{x}(Y \circ F)-\chi^{-1} G_{*}\left(\operatorname{div}_{x} Y\right)=\sum_{i=1}^{k}\left\langle\left(\nabla_{y} Y_{i}\right) \circ F, \frac{\partial}{\partial x_{i}} F_{x}\right\rangle d m
$$

as measures, where $\chi=d G_{*}(m) / d m$. In case $\operatorname{div}_{x} Y \in L_{\mathrm{loc}}^{1}$,

$$
\operatorname{div}_{x}(Y \circ F)-\left(\operatorname{div}_{x} Y\right) \circ F=\sum_{i=1}^{k}\left\langle\left(\nabla_{y} Y_{i}\right) \circ F, \frac{\partial F_{x}}{\partial x_{i}}\right\rangle,
$$

as functions, a.e.

The proofs of Proposition 4.8 and Lemmas 4.9, 4.10 are delayed to $\S 5$.

Proof of Lemma 4.6(a). It is well known that under the assumptions of Proposition 4.2

$$
g(x, \chi)=f \circ F(x, \eta)-\left\langle\eta, F_{x}(\eta)\right\rangle .
$$

By the assumptions and by Lemma $0.12, \nabla f \in L_{\mathrm{loc}}^{2}$ and $D f \in L_{\mathrm{loc}}^{2}$. Thus, the chain rule (4.18) is applicable and yields

$$
\frac{\partial g}{\partial x_{i}}(x, \eta)=\frac{\partial f}{\partial x_{i}} \circ F(x, \eta)+\sum_{j=1}^{N}\left(\frac{\partial f}{\partial y_{j}} \circ F(x, \eta)-\eta_{j}\right) \frac{\partial F}{\partial x_{i}}(x, \eta) \text { a.e., }
$$

where $F(x, \eta)=\left(x, F_{x}(\eta)\right)=\left(x, F_{1}(x, \eta), \ldots, F_{N}(x, \eta)\right)$. Since $G(F(x, \eta))$ $=(x, \eta),\left(\partial f / \partial y_{j}\right) \circ F(x, \eta)-\eta_{j}=0, j=1, \ldots, N$, and so

$$
\nabla_{x} g=\left(\nabla_{x} f\right) \circ F \quad \text { a.e., and } \nabla_{x} g \in L_{\text {loc }}^{2} \text {. }
$$

Proceeding in like manner (or using the standard facts on the Fenchel conjugate), we get

$$
\nabla_{\eta} g(x, \eta)=-F_{x}(x, \eta), \quad(x, \eta) \in \Omega \times R^{N} .
$$

In our notation the (distributional) Jacobian matrix of $G$ is

$$
D G=\left(\begin{array}{cc}
I & 0 \\
B_{21} & B_{22}
\end{array}\right) \text {. }
$$


By Lemma 0.12 , the (distributional) Jacobian matrix of $F=G^{-1}$ is represented by an $L_{\text {loc }}^{2}$ matrix-valued function which is

$$
D F=\left(\begin{array}{cc}
I & 0 \\
-B_{22}^{-1} B_{21} & B_{22}^{-1}
\end{array}\right) \circ F .
$$

This and (4.22) yield (4.14). By (4.21) $\widetilde{B}_{21}=\nabla_{x} \nabla_{\eta} g(x, \eta)=-\nabla_{x} F_{x}(x, \eta)=$ $-\left(B_{22}^{-1} B_{21}\right) \circ F(x, \eta)$ a.e., by (4.23). By symmetry, $\widetilde{B}_{12}=-\left(B_{12} B_{22}^{-1}\right) \circ F(x, \eta)$.

To obtain (4.16), we apply Lemma 4.10 to vector field $Y=\nabla_{x} f \in L_{\text {loc }}^{2}$. Clearly, $\operatorname{div}_{x} Y=\operatorname{tr} B_{11}$ is a Radon measure by the assumptions. On the other hand, by (4.21), $\operatorname{tr} \widetilde{B}_{11}=\operatorname{div}_{x} \nabla_{x} g=\operatorname{div}_{x}(Y \circ F)$. By Lemma 4.10, $\operatorname{tr} \widetilde{B}_{11}$ is a Radon measure and (4.19) becomes

$$
\operatorname{tr} \widetilde{B}_{11}-\chi^{-1} G_{*}\left(\operatorname{tr} B_{11}\right)=\sum_{i=1}^{k}\left\langle\left(\nabla_{y} Y_{i}\right) \cdot F, \frac{\partial}{\partial x_{i}} F_{x}\right\rangle d m .
$$

Since $\nabla_{y} Y_{i}$ is the $i$ th row of $B_{12}$ and, by (4.23), $\partial F_{x} / \partial x_{i}$ is the $i$ th column of $-B_{22} B_{21}$, (4.16) follows.

(b) If $\operatorname{tr} H_{11}$ is represented by an $L_{\text {loc }}^{1}$ function, then both $\operatorname{tr} B_{11}=4 \operatorname{tr} H_{11}$ and $\operatorname{tr} \widetilde{B}_{11}=4 \operatorname{tr} \widetilde{H}_{11}$ are $L_{\text {loc }}^{1}$ functions, by (4.16), which can now be written as $\operatorname{tr} \widetilde{B}_{11}=\left(\operatorname{tr} B_{11}\right) \circ F-\operatorname{tr}\left(B_{12} B_{22}^{-1} B_{21}\right) \circ F$, a.e. in $\Omega \times R^{N}$ (where all terms are now functions). By this

$$
\begin{aligned}
\operatorname{tr} \widetilde{H}_{11} & -\operatorname{tr} \widetilde{H}_{12} \widetilde{H}_{22}^{-1} \widetilde{H}_{21}=\frac{1}{4} \operatorname{tr} B_{11} \circ F \\
& -\frac{1}{4} \operatorname{tr}\left(B_{12} \circ F\right)\left(B_{22} \circ F\right)^{-1}\left(B_{21} \circ F\right)-\operatorname{tr} \widetilde{H}_{12} \widetilde{H}_{22}^{-1} \widetilde{H}_{21} \\
& =\left(\operatorname{tr} H_{11}\right) \circ F+\left(\operatorname{tr} \widetilde{H}_{0}-\operatorname{tr} \widetilde{H}_{12} \widetilde{H}_{22}^{-1} \widetilde{H}_{21}\right),
\end{aligned}
$$

where the equations hold a.e. and $\widetilde{H}_{0}$ denotes the Hermitian part of $-\frac{1}{2} B_{12}$ 。 $F(z, \xi)\left(B_{22} \circ F(z, \xi)\right)^{-1}\left(B_{21} \circ F\right)(z, \xi)$. Hence $\widetilde{H}_{22}(z, \xi), \widetilde{H}_{12}(z, \xi), \widetilde{H}_{21}(z, \xi)$ are Hermitian parts of

$$
-\frac{1}{2}\left(B_{22} \circ F\right)^{-1}, \quad \frac{1}{2}\left(B_{12} \circ F\right)\left(B_{22} \circ F\right)^{-1}, \quad \frac{1}{2}\left(B_{22} \circ F\right)^{-1}\left(B_{21} \circ F\right),
$$

by (4.14) and (4.15), we can apply Lemma 3.1 pointwise a.e. and get $\operatorname{tr} \widetilde{H}_{0}-$ $\operatorname{tr} \widetilde{H}_{12} \widetilde{H}_{22}^{-1} \widetilde{H}_{21}=-\operatorname{tr}\left(H_{12} H_{22}^{-1} H_{21}\right) \circ F$, a.e. Substituting this in (4.24) yields (4.17). Q.E.D.

Proof of Theorem 0.10. Let $f(z, w)=\frac{1}{2}\|w\|_{z}^{2}$ and $g(z, w)=-\frac{1}{2}|\xi|_{z}^{2}$, where $|\cdot|_{z}$ denotes the dual norm to $\|\cdot\|_{z}, z \in G, w, \xi \in \mathbf{C}^{n}$. As is well known, the function $\frac{1}{2}|\cdot|_{z}^{2}$ is the Fenchel dual to $\frac{1}{2}\|\cdot\|_{z}^{2}$ (apply, e.g., Asplund [1, Lemma 2] to $\left.\gamma(t)=\frac{1}{2} t^{2}\right)$, and so (4.13) holds. By Characterization 0.6 , both $f$ and $(-g)$ lie in $P_{\text {subh }}$, and so $\operatorname{tr} H_{11} f=\frac{1}{4} \operatorname{tr} B_{11}$ and $\operatorname{tr} H_{11}(-g)=-\frac{1}{4} \operatorname{tr} \widetilde{B}_{11}$ are nonnegative Radon measures. Since $\left(-\operatorname{tr} \widetilde{B}_{11}\right)+\chi^{-1} G_{*}\left(\operatorname{tr} B_{11}\right)$ is the sum of two nonnegative terms and, by (4.16), is absolutely continuous with respect to Lebesgue measure, 
each term is absolutely continuous, and so $\operatorname{tr} H_{11}, \operatorname{tr} \widetilde{H}_{11} \in L_{\text {loc }}^{1}$, and so (4.17) makes sense and holds. (Recall that functions $f$ and $g$ satisfy assumptions of Lemma 4.6; we continue to use notation of this lemma.) Since $f,(-g) \in P_{\text {subh }}$, by Lemma 4.4 the right-hand side of (4.17) is nonnegative, while the left-hand side is nonpositive a.e.; thus, both are zero a.e., that is, $\operatorname{tr} H_{11}-\operatorname{tr} H_{12} H_{22}^{-1} H_{21}=$ 0 . (Note that $\widetilde{H}_{i j}=-H_{i j}(-g)$.) Q.E.D.

Remark. Careful examination of the last proof would show that Lemma 0.12 is actually not needed for the proof of Theorem 0.10 .

Proof of Theorem 0.11. By Lemma 4.6(a) and the preceding proof, function $-g(z, \xi)=\frac{1}{2}|\xi|_{z}^{2}$ satisfies assumptions of Lemma 4.4(c). Since $\operatorname{tr} H_{11}$ $\operatorname{tr} H_{12} H_{22}^{-1} H_{21}=0$ a.e., (4.17) implies that

$$
\operatorname{tr} H_{11}(-g)-\operatorname{tr} H_{12}(-g) H_{22}(-g)^{-1} H_{21}(-g) \geq 0,
$$

and so $-g \in P_{\text {subh }}$, by Lemma 4.4(c). By the same lemma, $f \in P_{\text {subh }}$, and so $\left(\mathbf{C}^{n},\|\cdot\|_{z}\right)_{z \in G}$ is a harmonic interpolation family by Characterization 0.6 . Q.E.D.

\section{TECHNICAL PROOFS}

This section contains proofs of Proposition 4.8 and Lemmas 4.9, 4.10, and 0.12 .

Proof of Proposition 4.8 (sketch). If $E \subset \Omega \times R^{N}$ is compact, represent

$$
E=\bigcup_{x}\{x\} \times E_{x}, \quad E_{x} \subset R^{N} .
$$

If $K$ is the (uniform) Lipschitz constant for $F_{x}, F_{x}^{-1}$, then

$$
\left.K^{-N} m_{N}\left(E_{x}\right) \leq m_{N}\left(F_{x}\right)\right) \leq K^{N} m_{N}\left(E_{x}\right) .
$$

By (5.1), (5.2), and the Fubini theorem $K^{-N} m(E) \leq m(F(E)) \leq K^{N} m(E)$. Q.E.D.

Proof of Lemma 4.9 (sketch). (a) Since $m_{1}$ and $F \circ m$ are mutually absolutely continuous, $u \circ F$ is measurable and its equivalence class depends only on the class of $u$. Let $E$ be a relatively compact subset of $G_{1}$ and let $\rho(y)$ be the Radon-Nikodym derivative $d m / d\left(F m_{1}\right)$. Integration by substitution [7, $\left.\S 20.3\right]$ yields $\int_{E}|u \circ F(x)|^{p} \rho \circ F(x) d m_{1}=\int_{F(E)}|u(y)|^{p} d m$. Since $\varepsilon:=\inf$ ess $\rho \mid F(E)>$ 0 , we get $\|u \circ F\|_{L_{p}\left(E, m_{1}\right)} \leq \varepsilon^{1 / p}\|u\|_{L^{p}(F(E), m)}$, which proves (a) and implies (b).

(c) By [10, §1.15, Theorem 1], there exist smooth functions $u_{k} \in \mathbf{C}^{\infty}(G)$, $k=1,2, \ldots$, such that $u_{k} \rightarrow u$ in $L_{\mathrm{loc}}^{1}(G)$ and $\partial u_{k} / \partial y_{j} \rightarrow \partial u / \partial y_{j}$ in $L_{\mathrm{loc}}^{2}(G)$ for $j=1, \ldots, n$. A result of $\mathrm{Vol}^{\prime}$ pert [20, Theorem 13.2] implies that the 
distributional derivatives $\partial\left(u_{k} \circ F\right) / \partial x_{i}$ are represented by $L_{\text {loc }}^{1}\left(G_{1}\right)$ functions and, furthermore,

$$
\frac{\partial}{\partial x_{i}}\left(u_{k} \circ F\right)=\sum_{j=1}^{n}\left(\frac{\partial u_{k}}{\partial y_{j}}\right) \circ F \frac{\partial F_{j}}{\partial x_{i}} \quad \text { a.e. } d m_{1} .
$$

By (a), $u_{k} \circ F \in L_{\text {loc }}^{1}\left(G_{1}, m_{1}\right),\left(\partial u_{k} / \partial y_{j}\right) \circ F \in L_{\text {loc }}^{q}\left(G_{1}, m_{1}\right), k=1,2, \ldots$, $j=1, \ldots, n$. By (b), $u_{k} \circ F \rightarrow u \circ F$ in $L_{\text {loc }}^{1}$ and $\left(\partial u_{k} / \partial y_{j}\right) \circ F \rightarrow\left(\partial u / \partial y_{j}\right) \circ$ $F$ in $L_{\text {loc }}^{q}, j=1, \ldots, n$. Thus, the right-hand side of $(5.3)$ converges in $L_{\mathrm{loc}}^{1}\left(G_{1}, m_{1}\right)$ to $\sum_{j=1}^{k}\left(\partial u / \partial y_{j}\right) \circ F \partial F_{j} / \partial x_{i}$. The latter function represents $\partial(u \circ F) / \partial x_{i}$, because $\partial\left(u_{k} \circ F\right) / \partial x_{i} \rightarrow \partial(u \circ F) / \partial x_{i}$, as distributions. Q.E.D.

Proof of Lemma 4.10. Denote by $Y^{\varepsilon}, \varepsilon>0$, the standard convolution-regularizations of $Y$, as in $[10, \S 1.1 .5]$. Then $Y^{\varepsilon} \in \mathbf{C}^{\infty}\left(\Omega_{\varepsilon} \times R^{m}\right)$, where $\Omega_{\varepsilon}=\{x \in$ $\Omega: \operatorname{dist}(x, \partial \Omega)>\varepsilon\}$ and $Y^{\varepsilon} \rightarrow Y$ in $L_{\mathrm{loc}}^{1}, \partial Y^{\varepsilon} / \partial y_{j} \rightarrow \partial Y / \partial y_{j}$ in $L_{\mathrm{loc}}^{2}$, $j=1, \ldots, n$. By the chain rule $(4.18)$, we get

$$
\operatorname{div}_{x}\left(Y^{\varepsilon} \circ F\right)-\left(\operatorname{div}_{x} Y^{\varepsilon}\right) \circ F=\sum_{i=1}^{k}\left\langle\left(\nabla_{y} Y_{i}^{\varepsilon}\right) \circ F, \frac{\partial}{\partial x_{i}} F_{x}\right\rangle .
$$

By Lemma 4.9(a), (b), $Y \circ F \in L_{\text {loc }}^{1},\left(\nabla_{y} Y_{i}\right) \circ F \in L_{\text {loc }}^{2}$, and $Y^{\varepsilon} \circ F \rightarrow Y \circ F$ in $L_{\mathrm{loc}}^{1},\left(\nabla_{y} Y_{i}^{\varepsilon}\right) \circ F \rightarrow\left(\nabla_{y} Y_{i}\right) \circ F$ in $L_{\mathrm{loc}}^{2}$. Since $\partial F_{x} / \partial x_{i} \in L_{\mathrm{loc}}^{2}$, we obtain that (5.4)

$$
\operatorname{div}_{x}\left(Y^{\varepsilon} \circ F\right)-\left(\operatorname{div}_{x} Y^{\varepsilon}\right) \circ F \quad \text { converges in } L_{\text {loc }}^{1} \text { to } \sum_{i=1}^{k}\left\langle\left(\nabla_{y} Y\right) \circ F, \frac{\partial}{\partial x_{i}} F_{x}\right\rangle \text {. }
$$

Assertion. In the notation and under assumptions of Definition 4.7 and Lemma 4.9 , if $f_{n} \in L_{\mathrm{loc}}^{1}(G), n=1,2, \ldots$, and $f_{n} d m \rightarrow d \mu$ in weak convergence of measures (i.e., for every compactly supported function $g, \int g f_{n} d m \rightarrow$ $\left.\int g d \mu\right)$, then $\left(f_{n} \circ F\right) d m_{1}$ converges weakly to the measure $\left(1 / \chi_{0}\right) d\left(F^{-1}\right)_{*} \mu$, where $\chi_{0}$ is the Radon-Nikodym derivative $d\left(F^{-1}\right)_{*} m / d m_{1}$.

The assertion follows easily from standard theorems; see [8, $\S \S 12.45,20.3]$.

By the assertion, the measures $\left(\operatorname{div}_{x} Y^{\varepsilon}\right) \circ F d m$ converge weakly (as $\varepsilon \rightarrow 0$ ) to the Radon measure $(1 / \chi) G_{*}(\operatorname{div} Y)$, while $\operatorname{div}_{x}\left(Y^{\varepsilon} \circ F\right) \rightarrow \operatorname{div}_{x}(Y \circ F)$ in the topology of distributions on $\Omega \times R^{n}$. In view of these observations and (5.4), $\operatorname{div}_{x}(Y \circ F)$ is represented by a Radon measure and

$$
\operatorname{div}_{x}(Y \circ F)-\chi^{-1} G_{*}\left(\operatorname{div}_{x} Y\right)=\sum_{i=1}^{k}\left\langle\left(\nabla_{y} Y\right) \circ F, \frac{\partial}{\partial x_{i}} F_{x}\right\rangle d m
$$

where $\chi=d G_{*}(m) / d m$.

Observe finally that, if $\operatorname{div}_{x} Y=k d m$, where $k \in L_{\text {loc }}^{1}$, then $\chi^{-1} G_{*}(k d m)$ $=(k \circ F) d m$. Then also $\operatorname{div}_{x}(Y \circ F)$ is represented by an $L_{\text {loc }}^{1}$ function. 
Then identifying $\operatorname{div}_{x} Y$ and $\operatorname{div}_{x}(Y \circ F)$ with corresponding functions, we can rewrite $(5.5)$ as

$$
\operatorname{div}_{x}(Y \circ F)-\left(\operatorname{div}_{x} Y\right) \circ F=\sum_{i=1}^{k}\left\langle\left(\nabla_{y} Y\right) \cdot F, \frac{\partial}{\partial x_{i}} F_{x}\right\rangle \quad \text { a.e. Q.E.D. }
$$

The remainder of this section is devoted to the proof of Lemma 0.12. First, we make some rather obvious observations.

Remark 5.1. In the context of Lemma 0.12 , the following integration by substitution formula is valid:

$$
\int_{\Omega \times R^{n}} f\left(x^{\prime}, y^{\prime}\right) d x^{\prime} d y^{\prime}=\int_{\Omega \times R^{n}}(f \circ G)(x, y) w(x, y) d x d y
$$

for every $f \in L^{1}\left(\Omega \times R^{n}\right)$, where $w(x, y)$ is equal a.e. to the Jacobian determinant of $G$.

The proof follows from the fact that the Jacobian matrix of $G(x, y)=$ $\left(x, G_{x}(y)\right)$ has the block lower-triangular form relative to the decomposition $R^{k} \times R^{n}$, and the diagonal blocks are the identity matrix and the Jacobian matrix of the map $y \rightarrow G_{x}(y): R^{n} \rightarrow R^{n}$. Thus, $w(x, y)=J\left(G_{x}(y)\right)$ a.e. Since $G_{x}: R^{n} \rightarrow R^{n}$ is bi-Lipschitz, it is well known that

$$
\int_{R^{n}} f\left(x^{\prime}, y^{\prime}\right) d y^{\prime}=\int_{R^{n}} f\left(x^{\prime}, G_{x}(y)\right) J\left(G_{x}(y)\right) d y \text { a.e. }
$$

(with $x=x^{\prime}$ here). Integration with respect to $d x^{\prime}$ yields the desired formula.

Remark 5.2. The inverse of the Jacobian matrix of $G$ has the block lowertriangular form with respect to the decomposition of $R^{k} \times R^{n}$, namely

$$
(D G)(x, y)^{-1}=\left(\begin{array}{cc}
I & 0 \\
-\left(\mathbf{C}^{-1} B\right)(x, y) & \mathbf{C}(x, y)^{-1}
\end{array}\right),
$$

where $I, 0, B(x, y), \mathbf{C}(x, y)$ are the blocks of $(D G)(x, y)$, of sizes $k \times k$, $k \times n, n \times k$, and $n \times n$, respectively. Since $G_{x}$ is bi-Lipschitz (locally uniformly in $x), \mathbf{C}, \mathbf{C}^{-1} \in L_{\mathrm{loc}}^{\infty}$, and since $B \in L_{\mathrm{loc}}^{2}$, by assumptions, $\left(-\mathbf{C}^{-1} B\right) \in L_{\mathrm{loc}}^{2}$.

Proof of Lemma 4.13 (sketch). To make the argument more transparent, we will employ the specific structure of our maps $G, F$ only at the end of the proof. Thus, at the beginning we assume only that $G$ is a homeomorphism of $\Omega_{1}$ onto $\Omega$, where $\Omega_{1}, \Omega \subset R^{N}$, with the inverse $F(v)=u$. (Eventually, we will let $R^{N}=R^{k} \times R^{n}, u=(x, y)$.) We assume also that the distributional Jacobian matrix is represented by a locally square-integrable matrix-valued function, denoted $(D G)(u)$, whose determinant $w(u)$ is positive a.e. Assume further that $w, w^{-1} \in L_{\mathrm{loc}}^{\infty}\left(\Omega_{1}\right)$ and that the formula

$$
\int_{\Omega} f(v) d v=\int_{\Omega_{1}}(f \circ G)(u) w(u) d u
$$


holds for $f \in L_{0}^{1}(\Omega) \quad\left(=L^{1}\right.$ functions with compact support in $\Omega$ ). Assume, furthermore, that the matrix-valued function $T(v)=[(D G)(F v)]^{-1}$ is locally square-integrable. (All this holds by Remarks 5.1, 5.2, and Lemma 4.9.)

We intend to show that the matrix-valued function $T(\cdot)$ actually represents the distributional Jacobian matrix of $F$. The necessary and sufficient condition for this is that the vector equation

$$
\int T(v) g(v) d v=-\int(\operatorname{div} g) F(v) d v
$$

holds for all $g=\left(g_{1}, \ldots, g_{N}\right) \in \mathbf{C}_{0}^{\infty}(\Omega)$. in order to prove this identity, we will first reduce it to a statement on $G(\cdot)$.

Substituting $v=G(u)$ in (5.8), we obtain, by (5.7), that Eq. (5.8) is equivalent to

$$
\int T(G(u))(g \circ G)(u) w(u) d u=-\int(\operatorname{div} g) \circ G(u) F \circ G(u) d u .
$$

Denote $\rho(u)=(g \circ G)(u)$. Clearly, $\rho \in \mathbf{C}_{0}\left(\Omega_{1}\right)$ (= continuous functions on $R^{N}$ with compact support in $\Omega_{1}$ ) and, by Lemma 4.9 (in particular, the chain rule (4.18)), $D \rho \in L^{2}$. Furthermore, $F \circ G(u)=u, T(G u)=(D G)(u)^{-1}$, and one can easily compute that $\operatorname{div} g(G(u))=\operatorname{tr}\left[(D \rho)(u)(D G)(u)^{-1}\right]$. Now let $A(u)=\left(A_{i j}(u)\right)$ denote the transpose of the adjoint matrix of $(D G)(u)$, i.e., $A_{i j}(u)=(-1)^{i+j} M_{j i}(u)$, where $M_{i j}(u)$ is the $i j$ minor of $(D G)(u)$. Then $w(u)(D G)(u)^{-1}=A(u)$. Applying all these observations to (5.9), we obtain that $(5.8)$ is equivalent to the vector equation

$$
\int_{\Omega}(A(u) \rho(u)+[\operatorname{tr}(D \rho)(u) A(u)] u) d u=0
$$

(As already observed, $\rho$ has compact support and $D \rho \in L_{\text {loc }}^{2}$; furthermore, $A \in L_{\text {loc }}^{2}$. Hence, the integral makes sense.)

Denote by $\alpha$ the exterior differential form

$$
\alpha=(A(u) \rho(u)+[\operatorname{tr}(D \rho)(u) A(u)] u) d u_{1} \wedge \cdots \wedge d u_{N} .
$$

We have to show that $\int_{\Omega} \alpha=0$, which we will do by constructing a sequence of smooth and exact forms $\alpha_{\varepsilon(n)}$, with compact support in $\Omega$, so that the coefficient functions of $\alpha_{\varepsilon(n)}$ converge to those of $\alpha$, in the weak-star topology of $L^{1}(\Omega)$.

Let $G^{\varepsilon}, \varepsilon>0$, be the standard regularizations of the vector-valued function $G$ (by convolution with smooth kernels of small support; see [10, §1.1.5]). Fix $E$, a compact subset of $\Omega \times R^{n}$ (we will now use the special features of the map $G$, in the setting $\left.R^{N}=R^{k} \times R^{n}\right)$, such that $\operatorname{supp} \rho \subset \operatorname{Int}(E)$. Then $G^{\varepsilon} \rightarrow G$ in $L^{1}(E)$. Furthermore, $\partial\left(G_{i}^{\varepsilon}\right) / \partial u_{j}=\left(\partial G_{i} / \partial u_{j}\right)^{\varepsilon}$, and for each pair of indices $i j$, functions $\partial\left(G_{i}^{\varepsilon}\right) / \partial u_{j}$ converge to $\partial G_{i} / \partial u_{j}$ in every $L^{p}(E)$ space to which 
$\partial G_{i} / \partial u_{j}$ belongs, provided $1 \leq p<+\infty$. In particular,

$$
\frac{\partial}{\partial x_{i}}\left[G_{x}(y)\right]^{\varepsilon} \rightarrow \frac{\partial}{\partial x_{i}} G_{x}(y) \text { in } L^{2}(E)
$$

On the other hand, since $\partial G_{x}(y) / \partial y_{j} \in L^{\infty}(E)$, the approximations $\partial\left[G_{x}(y)\right]^{\varepsilon} / \partial y_{j}, \varepsilon>0$, form a bounded subset of $L^{\infty}(E)$, which also forms a norm-convergent net, to $\partial G_{x}(y) / \partial y_{i}$, in $L^{p}(E)$, for every $p \in(1,+\infty)$. Using the proof of [8, Theorem (13.11)], we can select a sequence $\varepsilon(s) \searrow 0$, $s=1,2, \ldots$ (which we fix for the rest of the proof) such that for $j=1, \ldots, n$

$$
\frac{\partial}{\partial y_{j}}\left[G_{x}(y)^{\varepsilon(s)}\right] \rightarrow \frac{\partial}{\partial y_{j}} G_{x}(y), \quad \text { a.e. on } E \text {. }
$$

Note, finally, that the $k \times k$ block of the matrix $D\left(G^{\varepsilon}\right)$ is the identity matrix, while the $k \times n$ block is the zero matrix.

Now denote by $A_{\varepsilon}(u)$, where $u=(x, y)$, the transpose of the adjoint matrix to $D\left(G^{\varepsilon}\right)(u)$ and let

$$
\alpha_{\varepsilon}=\left\{A_{\varepsilon}(u) \rho^{\varepsilon}(u)+\left[\operatorname{tr}\left(D \rho^{\varepsilon}\right)(u) A_{\varepsilon}(u)\right] u\right\} d u_{1} \wedge \cdots \wedge d u_{N},
$$

where $\rho^{\varepsilon}(u)$ is the convolution-regularization of $u$. (Note, however, that $A_{\varepsilon}(\cdot)$ is not a convolution of $A(\cdot)$ with a smooth kernel.) We will show now that $\alpha_{\varepsilon(s)}, s=1,2, \ldots$, converge to $\alpha$ in the weak-star topology of the space of forms with coefficients in $L^{1}(E)$. It is the only part of the proof in which we have to utilize the special properties of the map $G(x, y)=\left(x, G_{x}(y)\right)$.

By writing down in the standard way each minor $M_{i j, \varepsilon(s)}$ of $A_{\varepsilon(s)}$ as the sum of $(N-1)$ ! products, each consisting of $N-1$ factors, we represent each coefficient function of $\alpha_{\varepsilon(s)}$ (and parallely those of $\alpha$ ) as a sum of some products. If we discount all those zero products which contain at least one of the zero entries of the $k \times n$ block of the matrix $D\left[G(u)^{\varepsilon(s)}\right]($ or $D G(u))$, the remaining products are of the following two kinds:

First kind contains at most one term $\partial\left[G_{x}^{j}(y)^{\varepsilon(s)}\right] / \partial x_{i}, \quad i=1, \ldots, k$, $j=1, \ldots, n$, one term $\rho_{p}(x, y)^{\varepsilon(s)}$, and several terms of the form $\partial\left[G_{x}^{q}(y)^{\varepsilon(s)}\right] / \partial y_{j}$.

Second kind is similar, but factor $\rho_{p}(x, y)^{\varepsilon(s)}$ is replaced by $\partial \rho_{p}^{\varepsilon(s)} / \partial u_{q}$ $\left(u_{q}=x_{i}\right.$ or $\left.y_{i}\right)$ and factor $u_{r}$ is added.

We now claim that each such product in $\alpha$ is the limit, in the weak-star topology of $L^{1}(E)$, of the corresponding products in $\alpha_{\varepsilon(s)}, s=1,2, \ldots$. The following observations make it clear.

(a) Since $\rho^{\varepsilon} \rightarrow \rho$ uniformly on $E$ and $\partial\left(\rho^{\varepsilon}\right) / \partial u_{j} \rightarrow \partial \rho / \partial u_{j}$ in $L^{2}(E)$, and by (5.12), we obtain that both

$$
\frac{\partial}{\partial x_{i}} G_{x}^{j}(y)^{\varepsilon(s)} \cdot \rho_{p}^{\varepsilon(s)} \rightarrow \frac{\partial}{\partial x_{i}} G_{x}^{j}(y) \cdot \rho_{p} \quad \text { in } L^{1}(E)
$$


and

$$
\frac{\partial}{\partial x_{i}} G_{x}^{j}(y)^{\varepsilon(s)} \cdot \frac{\partial}{\partial u_{q}} \rho_{p}^{\varepsilon(s)} \rightarrow \frac{\partial}{\partial x_{i}} G_{x}(y) \cdot \frac{\partial}{\partial u_{q}} \rho_{p} \quad \text { in } L^{1}(E) .
$$

(b) The product of several uniformly bounded, pointwise a.e. convergent sequences of functions on $E$, (e.g., of the form (5.13)) is a uniformly bounded, pointwise a.e. convergent sequence of functions on $E$.

(c) By (a) and (b), either of the products of first or second kind in $\alpha_{\varepsilon(s)}$, $s=1,2, \ldots$, or $\alpha$, is now reduced to the form $a_{s}(u) b_{s}(u), u=1,2, \ldots$, or $a(u) b(u), u \in E$, where

$$
\begin{gathered}
\sup _{s}\left\|a_{s}\right\|_{\infty} \leq \mathbf{C}<+\infty, \quad a_{s}(u) \rightarrow a(u) \quad \text { a.e. on } E ; \\
\left\|b_{s}-b\right\|_{1} \rightarrow 0 \quad\left(\text { in } L^{1}(E)\right) .
\end{gathered}
$$

The proof of the next assertion is an easy exercise.

Assertion If (5.14), (5.15) hold, then $a_{s} b_{s} \rightarrow a b$ weak-star in $L^{1}(E)$.

Thus, $\alpha_{\varepsilon(s)} \rightarrow \alpha$ weak-star, as required.

It remains to check that every $\alpha_{\varepsilon(s)}, s=1,2, \ldots$, is an exact form. Without loss of generality, we can just show that the form $\alpha$, defined by $(5.11)$, is exact, provided the maps $G$ and $\rho$ are smooth. It turns out that $\alpha=d \beta$, where

$$
\beta=\sum_{i j=1}^{N}(-1)^{j+1} M_{j i}(u) \rho_{j}(u) u d u_{1} \wedge \cdots \wedge \hat{d} u_{i} \wedge \cdots \wedge d u_{N}
$$

(Note that $\beta$ is vector-valued; it has compact support because $\rho$ does.) Exterior differentiation yields easily that $\alpha=d \beta+\lambda$, where

$$
\lambda=\sum_{i j}(-1)^{i+j} \frac{\partial}{\partial u_{i}}\left(M_{j i}\right) \rho_{j}(u) d u_{1} \wedge \cdots \wedge d u_{N} .
$$

The proof is concluded by observing that $\lambda=0$, as a direct consequence of the identities

$$
\sum_{j=1}^{N}(-1)^{i} \frac{\partial}{\partial u_{j}} M_{j i}=0, \quad i=1,2, \ldots, N .
$$

To prove these is an exercise in the algebra of determinants. Q.E.D.

\section{REFERENCES}

1. E. Asplund, Frechet differentiability of convex functions, Acta Math. 121 (1968), 31-48.

2. E. Bedford, B. A. Taylor, The Dirichlet problem for a complex Monge-Ampere equation, Invent. Math. 37 (1976), 1-44.

3. R. Coifman, M. Cwikel, R. Rochberg, Y. Sagher, and G. Weiss, The complex method for interpolation of operators acting on families of Banach spaces, Lecture Notes in Math., vol. 779, Springer-Verlag, Berlin and New York, 1980, pp. 123-153.

4. $\ldots$ A theory of complex interpolation for families of Banach spaces, Adv. in Math. 33 (1982), 203-229. 
5. R. Coifman and S. Semmes, Interpolation of Banach spaces and nonlinear Dirichlet problems, Lecture Notes in Math., vol. 1302, Springer-Verlag, Berlin and New York, 1988.

6. M. M. Day, Normed linear spaces, Springer-Verlag, New York, 1973.

7. W. K. Hayman and P. B. Kennedy, Subharmonic functions, Vol. I, Academic Press, London, 1976.

8. E. Hewitt and K. Stromberg, Real and abstract analysis, Springer-Verlag, New York, 1969.

9. C. O. Kiselman, The partial Legendre transformation for plurisubharmonic functions, Invent. Math. 49 (1978), 137-148.

10. V. G. Mazja, Sobolew spaces, Springer-Verlag, Berlin, 1985.

11. R. Rochberg, The work of Coifman and Semmes on complex interpolation, several complex variables and PDE's, U.S.-Swedish Seminar on Function Spaces and Applications, Lund, June 1986 (to appear).

12. __ Interpolation of Banach spaces and negatively curved vector bundles, Pacific J. Math. 110 (1984), 335-376.

13. R. Rochberg and G. Weiss, Some topics in complex interpolation theory, Topics in Modern Harmonic Analysis, Istituto Nazionale di Alta Matematica, Rome, 1983, pp. 769-818.

14a. Z. Slodkowski, Analytic multifunctions and their applications, talk at the Banach Space Conference, Kent, August 1985.

14b. _ Complex interpolation families of normed spaces over several-dimensional parameter space, Abstracts of the Special Session in Several Complex Variables, 826th Meeting of the AMS, Indianapolis, April 1986.

14c. __ On complex interpolation methods for families of normed spaces over domains in $C^{k}$, talk at the International Conference on Harmonic Measure, Toledo, Ohio, July 1986.

15. __ Local maximum property and q-plurisubharmonic functions in uniform algebras, J. Math. Anal. Appl. 115 (1986), 105-130.

16. __ Pseudoconvex classes of functions. I. Pseudoconcave and pseudoconvex sets, Pacific J. Math. 134 (1988), 343-376.

17. __ Complex interpolation of normed and quasinormed spaces. I, Trans. Amer. Math. Soc. 308 (1988), 685-711.

18. __ Pseudoconvex classes of functions. III. Characterization of dual pseudoconvex classes on complex homogeneous spaces, Trans. Amer. Math. Soc. 309 (1988), 165-189.

19. __ Complex interpolation of normed and quasinormed spaces in several dimensions. II. Properties of harmonic interpolation, Trans. Amer. Math. Soc. 317 (1990), 255-285.

20. A. I. Vol' pert, The spaces $B V$ and quasilinear equations, Math. USSR-Sb. 2 (1967), 225-267.

21. S. Kobayashi, Negative vector bundles and complex Finsler structures, Nagoya Math. J. 57 (1975), 153-166.

22. R. Coifman and S. Semmes, Interpolation of Banach spaces, Perron processes and Yang-Mills.

23. S. Semmes, Interpolation of Banach spaces, differential geometry and differential equations, preprint.

Department of Mathematics, University of Illinois, Chicago, Illinois 60680 